\title{
Interferon- $\gamma$ converts human microvascular pericytes into negative regulators of alloimmunity through induction of indoleamine 2,3-dioxygenase 1
}

\author{
Rebecca Liu, ${ }^{1}$ Jonathan Merola, ${ }^{2}$ Thomas D. Manes, ${ }^{1}$ Lingfeng Qin, ${ }^{2}$ Gregory T. Tietjen, ${ }^{2}$ \\ Francesc López-Giráldez, ${ }^{3}$ Verena Broecker, ${ }^{4}$ Caodi Fang, ${ }^{5}$ Catherine Xie, ${ }^{1}$ Ping-Min Chen, ${ }^{1}$ \\ Nancy C. Kirkiles-Smith, ${ }^{1}$ Dan Jane-Wit, ${ }^{5}$ and Jordan S. Pober ${ }^{1}$ \\ 'Department of Immunobiology, and 'Department of Surgery, Yale School of Medicine, New Haven, Connecticut, USA. \\ ${ }^{3}$ Yale Center for Genome Analysis, Yale University, New Haven, Connecticut, USA. ${ }^{4}$ Department of Histopathology, \\ Addenbrooke's Hospital, Cambridge University Hospitals, Cambridge, United Kingdom. ${ }^{5}$ Section of Cardiovascular \\ Medicine, Yale School of Medicine, New Haven, Connecticut, USA.
}

Early acute rejection of human allografts is mediated by circulating alloreactive host effector memory $T$ cells $\left(T_{E M}\right) \cdot T_{E M}$ infiltration typically occurs across graft postcapillary venules and involves sequential interactions with graft-derived endothelial cells (ECs) and pericytes (PCs). While the role of ECs in allograft rejection has been extensively studied, contributions of PCs to this process are largely unknown. This study aimed to characterize the effects and mechanisms of interactions between human PCs and allogeneic $\mathrm{T}_{\mathrm{EM}}$. We report that unstimulated PCs, like ECs, can directly present alloantigen to $T_{E M}$, but while IFN- $\gamma$-activated ECs ( $\gamma$-ECs) show increased ability to stimulate alloreactive T cells, IFN- $\gamma$-activated PCs $\left(\gamma\right.$-PCs) instead suppress $T_{E M}$ proliferation but not cytokine production or signaling. RNA sequencing analysis of PCs, $\gamma$-PCs, ECs, and $\gamma$-ECs reveal induction of indoleamine 2,3-dioxygenase 1 (IDO1) in $\gamma$-PCs to significantly higher levels than in $\gamma$-ECs that correlates with tryptophan depletion in vitro. Consistently, shRNA knockdown of ID01 markedly reduces $\gamma$-PC-mediated immunoregulatory effects. Furthermore, human PCs express ID01 in a skin allograft rejection humanized mouse model and in human renal allografts with acute T cell-mediated rejection. We conclude that immunosuppressive properties of human PCs are not intrinsic but instead result from IFN- $\gamma$-induced IDO1-mediated tryptophan depletion.

Conflict of interest: The authors have declared that no conflict of interest exists.

Submitted: October 4, 2017 Accepted: January 25, 2018 Published: March 8, 2018

Reference information: JCI Insight. 2018;3(5):e97881. https:// doi.org/10.1172/jci.insight.97881.

\section{Introduction}

Allogeneic transplantation is the most effective therapy for end-stage organ failure of the heart, kidney, lung, and liver, but despite improvements in immunosuppression, rejection of allogeneic organs by the host immune system remains a major contributor to poor graft outcome. Early acute rejection is generally a cellmediated event initiated by infiltration of circulating host $\mathrm{T}$ cells into the graft. In humans, the pretransplant frequency of circulating donor-reactive effector memory $\mathrm{T}$ cells $\left(\mathrm{T}_{\mathrm{EM}}\right)$, but not of total $\mathrm{T}$ cells, predicts likelihood and severity of posttransplant acute rejection events (1) and adoptively transferred human $\mathrm{T}_{\mathrm{EM}}$ are necessary and sufficient for rejection of allogeneic human skin grafts on immunodeficient mice (2). Such alloreactive $\mathrm{T}_{\mathrm{EM}}$ are generated in response to prior infections because their $\mathrm{T}$ cell receptors (TCRs) for antigen, engaged by self-allelic major histocompatibility complex (MHC) molecules displaying microbial peptides during the infectious episode, then cross-react with non-self allelic MHC molecules bearing a variety of peptides displayed on allogeneic graft cells, a process referred to as direct recognition of alloantigen (3). The direct allogeneic response is strong because many $\mathrm{T}$ cell clones are involved, but otherwise has the same requirements as responses to conventional antigens. Of relevance to transplantation, the activation of $\mathrm{T}_{\mathrm{EM}}$ by (allo)antigen may utilize different costimulators than those used by naive or central memory cells, allowing them to be activated by a somewhat wider variety of cell types acting as antigen-presenting cells (APCs). In humans, many of the costimulatory molecules utilized by $\mathrm{T}_{\mathrm{EM}}$ are displayed by the endothelial cells (ECs) lining peripheral blood vessels (4). Consequently, early rejection of human allografts can be mediated by 
host circulating $\mathrm{T}_{\mathrm{EM}}$ that directly recognize donor MHC class I and class II molecules expressed by graft ECs, explaining why elimination of professional APCs, such as dendritic cells, from the graft may reduce but is insufficient to prevent rejection (5). Human ECs may thus be classified as semiprofessional APCs because they have the capacity to reactivate resting alloreactive $\mathrm{T}_{\mathrm{EM}}$ to produce cytokines, to proliferate and to differentiate into effector cells, but cannot activate resting naive T cells (2).

Antigen presentation by ECs is distinct from presentation by other cell types because it also induces transendothelial migration of circulating $\mathrm{T}_{\mathrm{EM}}$, but not of naive $\mathrm{T}$ cells or central memory $\mathrm{T}$ cells, into tissues (6). In rejecting allografts, circulating host $\mathrm{T}_{\mathrm{EM}}$ must cross through the graft blood vessel walls to enter the parenchyma, a process that generally occurs at the postcapillary venules. However, the extravasation process does not stop with transendothelial migration. Structurally, the postcapillary venules are formed from 2 vascular cell types, ECs that line the lumen and a surrounding layer of pericytes (PCs) embedded in a shared basement membrane with ECs. It has recently been shown that PCs actively participate in the transmigration process of neutrophils, guiding the extravasating neutrophils through the basement membrane and attenuating the basement membrane at specific locations, facilitating neutrophil entry into the tissue interstitium (7). Newly extravasated neutrophils then dwell for extended periods in contact with PCs on the outer surface of the vessel, often migrating along the vessel length. While it is unknown if PCs play a similar role in $\mathrm{T}$ cell recruitment, in early stages of rejection, host $\mathrm{T}$ cells that have crossed through the postcapillary venules typically also accumulate in a perivascular distribution for a prolonged period of time before they further migrate into the graft parenchyma. Thus, PCs may also potentially influence effector functions of newly extravasated $\mathrm{T}$ cells through contact-dependent and/or paracrine signals.

In mice, tumor vessel PCs have been shown to negatively regulate $\mathrm{CD} 4^{+} \mathrm{T}$ cell activation and proliferation, and further induce T cell anergy (8). Less is known about the functions of PCs in the context of alloimmunity. We have used human PCs isolated and cultured from placental microvessels, in parallel with the use of human ECs cultured from umbilical vein, as a model system to study $\mathrm{T}$ cell interactions with the cells of microvessel walls. We previously reported both that IFN- $\gamma$ treatment induces expression of MHC class II molecules on cultured human PCs, similar to its actions on cultured human ECs, and that non-self alleles of class II MHC molecules are recognized by many of the same alloreactive $\mathrm{CD}^{+} \mathrm{T}$ cells as respond to non-self ECs cultured from the same donor as the PCs (9). However, in contrast to their response to allogeneic IFN- $\gamma$-activated ECs ( $\gamma$-ECs), resting human peripheral blood CD4 ${ }^{+} \mathrm{T}$ cells cocultured with IFN- $\gamma$-activated PCs ( $\gamma$-PCs) do not proliferate and become refractory to stimulation (9). Our new results reported here specifically focus on circulating $\mathrm{T}_{\mathrm{EM}}$ expressing CD4 or CD8 and reveal that the inhibitory effects of PCs are induced by IFN- $\gamma$, affect proliferation but not cytokine production, and largely depend on PC expression of indoleamine 2,3-dioxygenase 1 (IDO1).

\section{Results}

$\gamma$-PCs inhibit allogeneic human peripheral blood $T_{E M}$ proliferation. Direct recognition of alloantigen involves TCR engagement with non-self allelic forms of MHC molecules expressed on the surface of an allogeneic cell. Microvascular human ECs in situ express high levels of both MHC class I and class II molecules basally (i.e., in the absence of inflammation), but MHC class I molecule expression is greatly diminished and MHC class II molecule expression is completely lost in cell culture (10). It is not clear whether PCs also basally express MHC class II in the absence of inflammation in situ. Human IFN- $\gamma$ induces both MHC class I and class II molecule expression on cultured HUVECs to levels comparable to those expressed on microvascular ECs in situ and that IFN- $\gamma$ similarly induces cultured human placental microvascular PCs to express MHC class I and II molecules to nearly the same level as that expressed on IFN- $\gamma$-treated HUVECs (Supplemental Table 1; supplemental material available online with this article; https://doi.org/10.1172/ jci.insight.97881DS1) $(9,11,12)$. To assess the potential influence of PCs on $\mathrm{T}_{\mathrm{EM}}$ in allograft rejection, we first examined whether $\gamma$-PCs can activate allogeneic $C D 4^{+} \mathrm{T}_{\mathrm{EM}}$. As noted in the introduction, this subset contains the alloreactive $\mathrm{T}$ cells that mediate rejection, but $\mathrm{CD}^{+} \mathrm{T}_{\mathrm{EM}}$ constitute fewer than $10 \%$ of total circulating $\mathrm{CD}^{+} \mathrm{T}$ cells and their responses could therefore have been overlooked in our prior study using the total peripheral blood $\mathrm{CD}^{+} \mathrm{T}$ cell population (9). Within the $\mathrm{CD} 4^{+} \mathrm{T}_{\mathrm{EM}}$ population, only a minor fraction (about $0.5 \%$ on average) are alloreactive to any particular allograft donor, and this fraction is even more infrequent in the $\mathrm{CD} 8^{+} \mathrm{T}_{\mathrm{EM}}$ subset. As shown in Figure 1A, while $\gamma$-ECs showed increased ability to stimulate alloreactive $\mathrm{CD}^{+} \mathrm{T}_{\mathrm{EM}}$ in comparison with unstimulated ECs, MHC class II-expressing $\gamma$-PCs did not induce allogeneic $\mathrm{CD} 4^{+} \mathrm{T}_{\mathrm{EM}}$ to proliferate, extending the previous findings that $\gamma$-PCs poorly stimulate 
A

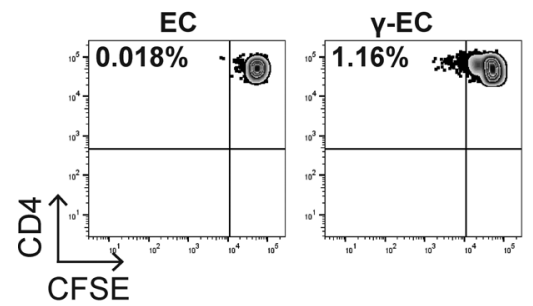

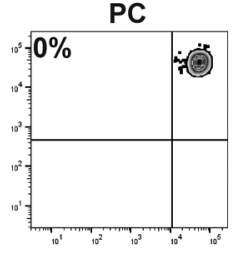

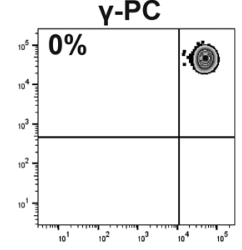

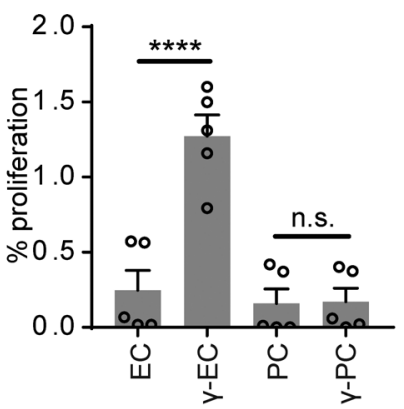

B
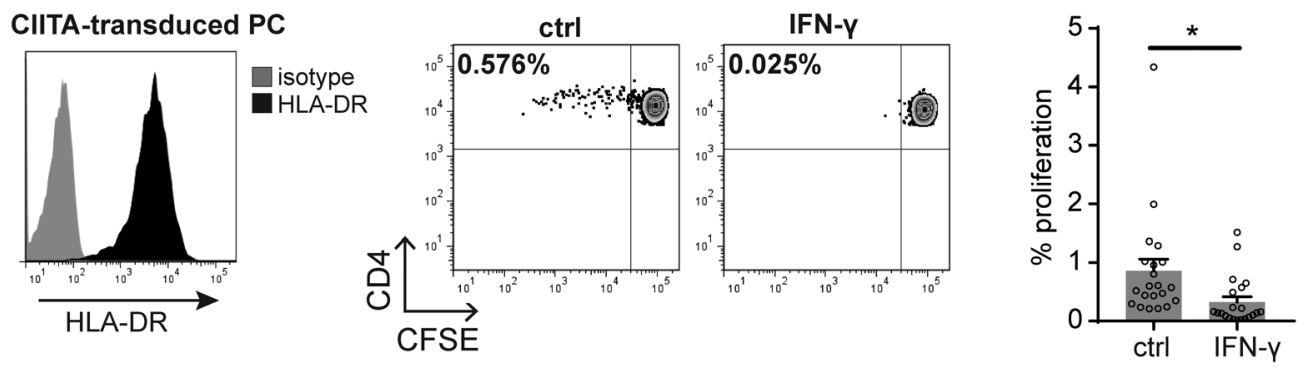

C

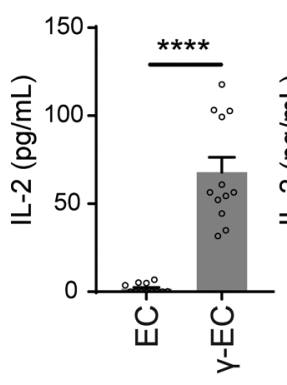

D

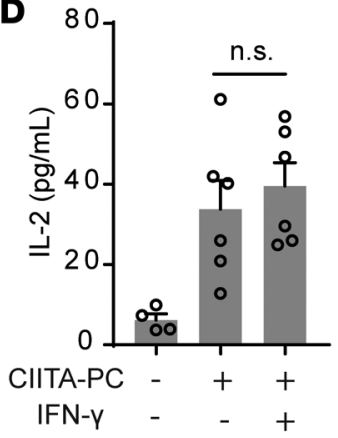

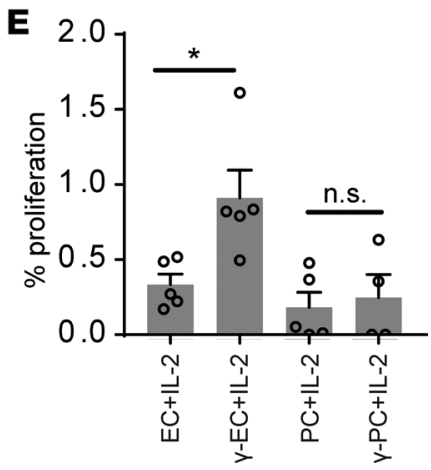

Figure 1. IFN- $\gamma$ activates PCs to potently inhibit $\mathrm{CD4}^{+} \mathrm{T}_{\mathrm{EM}}$ proliferation. (A) Proliferation of CFSE-labeled allogeneic CD4 ${ }^{+} \mathrm{T}_{\mathrm{EM}}$ cocultured with unstimulated endothelial cells (ECs), IFN- $\gamma$-activated ECs ( $\gamma$-ECs), unstimulated pericytes (PCs), or IFN- $\gamma$-activated PCs ( $\gamma$-PCs) for 7 days. ECs and PCs were isolated from syngeneic human umbilical cords and placentas, respectively ( $n=5$, one-way ANOVA, SEM). (B) Constitutive HLA-DR expression by PCs transduced with class II MHC transactivator (CIITA) (left). Proliferation of CFSE-labeled CD4 ${ }^{+} \mathrm{T}_{\mathrm{EM}}$ cocultured with unstimulated or IFN- $\gamma$-prestimulated CIITA-transduced PCs for 7 days (right) ( $n=21, t$ test, SEM). (C) ELISA measurement of IL-2 production by allogeneic CD4+ T $_{\text {EM }}$ cocultured with ECs, $\gamma$-ECs, PCs, or $\gamma$-PCs for 24 hours. ECs and PCs were isolated from syngeneic human umbilical cords and placentas, respectively $(n=12, t$ test, SEM). (D) ELISA measurement of IL-2 production by CD4 ${ }^{+} T_{E M}$ cocultured with unstimulated or IFN- $\gamma$-stimulated CIITA-transduced PCs for 24 hours $(n=4-6$, one-way ANOVA, SEM). (E) Proliferation of CFSE-labeled CD4 ${ }^{+} \mathrm{T}_{\mathrm{EM}}$ cocultured with ECs, $\gamma$-ECs, PCs, or $\gamma$-PCs. Recombinant IL- 2 was added ( $25 \mathrm{U} / \mathrm{ml}$ ) on day 3 of coculture, and CFSE dilution was assessed on day $7\left(n=4-5\right.$, one-way ANOVA). ${ }^{*} P<0.05,{ }^{* * *} P<0.0001$. n.s., not significant.

unfractionated circulating $\mathrm{CD} 4^{+} \mathrm{T}$ cells. We used IFN- $\gamma$ pretreatment of the PCs in this and prior experiments because expression of MHC class II molecules is required to engage the TCRs of the alloreactive T cell population. To determine if the behavior of $\gamma$-PCs is influenced by other IFN- $\gamma$-induced alterations in the PCs, we next performed coculture experiments using PCs transduced with class II MHC transactivator (CIITA-PCs) that constitutively express MHC class II molecules and related gene products, such as HLA$\mathrm{DM}$, but express few, if any, IFN- $\gamma$-induced genes other than those involved in MHC class II synthesis and expression (Figure 1B). We had previously shown that CIITA transduction in ECs can, like IFN- $\gamma$, induce MHC class II molecules and confer the ability to activate alloreactive CD4 ${ }^{+} \mathrm{T}$ cells (13), but this is the first time we have employed this approach in PCs. Strikingly, unstimulated CIITA-PCs induced robust CD4 ${ }^{+}$ $\mathrm{T}_{\mathrm{EM}}$ proliferation, but IFN- $\gamma$-pretreated CIITA-PCs significantly inhibited CD4 ${ }^{+} \mathrm{T}_{\mathrm{EM}}$ proliferation (Figure 1B). Treatment with IFN- $\gamma$ did not significantly alter the level of MHC class II expression by CIITA-PCs (Supplemental Figure 1), suggesting IFN- $\gamma$ reduces other costimulatory signals and/or induces an inhibitory mechanism(s) so that recognition of allogeneic MHC molecules on PCs by $\mathrm{CD}^{+} \mathrm{T}_{\mathrm{EM}}$ is no longer able to trigger proliferation.

Rapid proliferation and expansion of (allo)antigen-activated $\mathrm{T}$ cell populations require stimulation by 
A

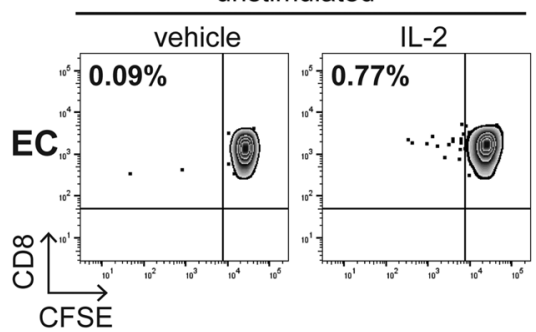

B

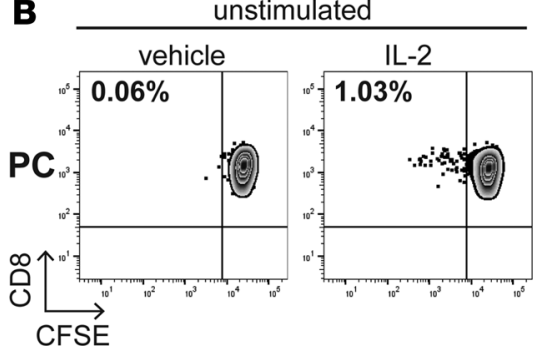

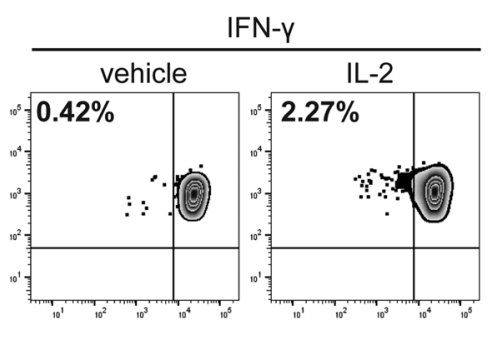
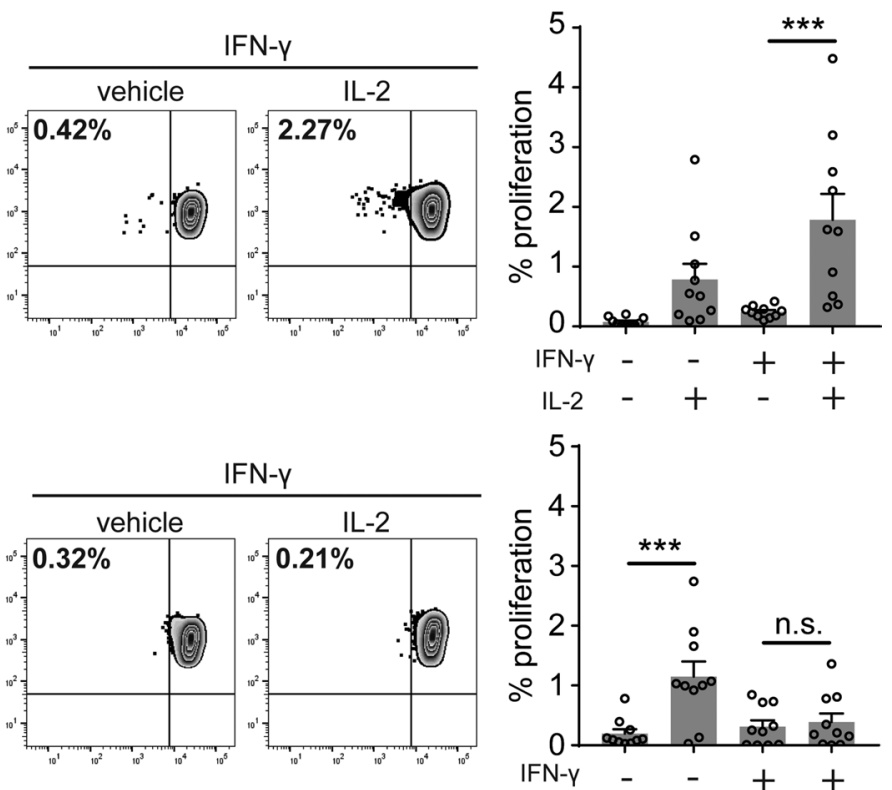

IFN-Y
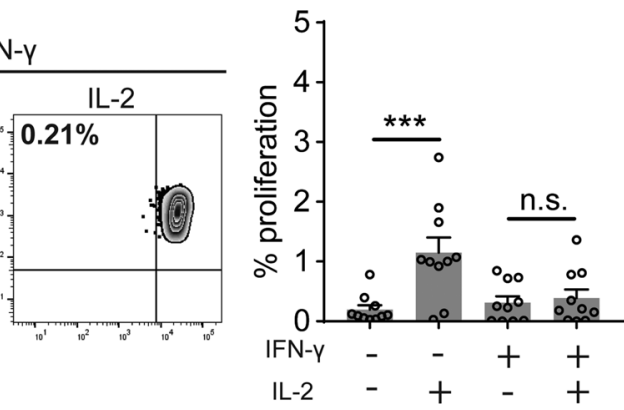

Figure 2. $\gamma$-PCs potently inhibit IL-2-induced $C D 8^{+} \mathbf{T}_{E M}$ proliferation. Proliferation of CFSE-labeled $C D 8^{+} T_{E M}$ cocultured with (A) unstimulated endothelial cells (ECs) or IFN- $\gamma$-activated ECs ( $\gamma$-ECs), or (B) unstimulated pericytes (PCs) or IFN- $\gamma$-activated PCs ( $\gamma$-PCs) for 7 days. Recombinant IL-2 (25 U/ml) was added on day 3 of coculture where indicated $\left(n=10\right.$, one-way ANOVA, SEM). ${ }^{* *} P<0.001$. n.s., not significant.

T cell growth factors that engage receptors that utilize the common $\gamma$ chain $\left(\gamma_{c}\right.$, designated as CD132). The principal such growth factor in vitro is IL-2, which is primarily made by activated $\mathrm{T}$ cells and serves as both an autocrine and paracrine growth factor. TCR recognition of antigen is required for acquisition of responsiveness of resting $\mathrm{T}_{\mathrm{EM}}$ cells to IL-2 because it induces the de novo expression of the IL-2R $\alpha$ chain (CD25), allowing binding of IL-2 to the signaling $\beta \gamma$ components of the IL-2R in the $10 \mathrm{pM}$ range, which is an approximately 100 -fold increase in sensitivity. We therefore examined whether the absence of proliferation we observed when $\mathrm{T}$ cells encountered $\gamma$-PCs was due to inhibition of IL-2 production by $\mathrm{CD} 4^{+} \mathrm{T}_{\mathrm{EM}}$. To assess this, allogeneic $\mathrm{CD} 4^{+} \mathrm{T}_{\mathrm{EM}}$ were cocultured with unstimulated or IFN- $\gamma$-pretreated ECs or PCs, and the level of IL-2 production by $\mathrm{T}_{\mathrm{EM}}$ was determined by ELISA. $\gamma$-PCs, like $\gamma$-ECs, activated CD $4^{+} \mathrm{T}_{\mathrm{EM}}$ to produce IL-2, though the level of production induced by $\gamma$-PCs was somewhat lower than that induced by $\gamma$-ECs (Figure 1C). More significantly, IFN- $\gamma$-stimulated CIITA-PCs induced a similar level of IL-2 production by $\mathrm{CD} 4^{+} \mathrm{T}_{\mathrm{EM}}$ as unstimulated CIITA-PCs (Figure 1D), suggesting that this difference between ECs and PCs was not related to the effects of IFN- $\gamma$ pretreatment. To confirm that the lack of proliferation in $\mathrm{CD} 4^{+} \mathrm{T}_{\mathrm{EM}}-\gamma-\mathrm{PC}$ coculture was not due to lower amounts of IL-2 produced, we supplemented exogenous recombinant IL-2 for $\mathrm{CD} 4^{+} \mathrm{T}_{\mathrm{EM}}$ cocultured with ECs and PCs. While addition of IL-2 enhanced CD4 ${ }^{+}$ $\mathrm{T}_{\mathrm{EM}}$ proliferation in response to stimulation by $\gamma$-ECs, it did not rescue the proliferative responses of $\mathrm{CD} 4^{+}$ $\mathrm{T}_{\mathrm{EM}}$ cultured with $\gamma$-PCs (Figure 1E). Collectively, these data suggest that the inhibition of proliferation by $\gamma$-PCs is not due to lack of IL-2 production.

Alloreactive human $\mathrm{CD} 8^{+} \mathrm{T}_{\mathrm{EM}}$ cells stimulated by allogeneic ECs can differentiate into cytotoxic $\mathrm{T}$ cells that are the primary effector population of early cell-mediated allograft rejection (14). $\mathrm{CD} 8^{+} \mathrm{T}_{\mathrm{EM}}$ are less efficient producers of IL-2 than $\mathrm{CD} 4^{+} \mathrm{T}_{\mathrm{EM}}$ and their full activation in vitro depends upon IL-2 provided by activated $C D 4^{+} \mathrm{T}_{\mathrm{EM}}$ (14). Potential interactions of $\mathrm{CD} 8^{+} \mathrm{T}_{\mathrm{EM}}$ with allogeneic PCs have not been previously examined. To determine if the inhibitory properties of $\gamma$-PCs also extend to $C D 8^{+} \mathrm{T}_{\mathrm{EM}}$, we conducted similar coculture experiments and assessed proliferation of $C D 8^{+} \mathrm{T}_{\mathrm{EM}}$ in response to same-donor ECs and PCs. Based on our previous experience (15), addition of exogenous recombinant IL-2 to cultures that lack CD4 ${ }^{+}$ $\mathrm{T}_{\mathrm{EM}}$ cells is necessary to induce proliferation except at very high $C D 8^{+} \mathrm{T}_{\mathrm{EM}}$ cell/stimulator cell ratios due to the limited extent of IL-2 produced by $\mathrm{CD} 8^{+} \mathrm{T}_{\mathrm{EM}}$ after activation. We found that ECs effectively stimulated $\mathrm{CD} 8^{+} \mathrm{T}_{\mathrm{EM}}$ proliferation in the presence of IL-2, and $\gamma$-ECs, expressing higher levels of MHC class I than unstimulated ECs, induced even higher levels of $C D 8^{+} T_{E M}$ proliferation (Figure 2A). While unstimulated PCs were fully capable of stimulating significant $C D 8^{+} \mathrm{T}_{\mathrm{EM}}$ cell proliferation in the presence of exogenous 
A

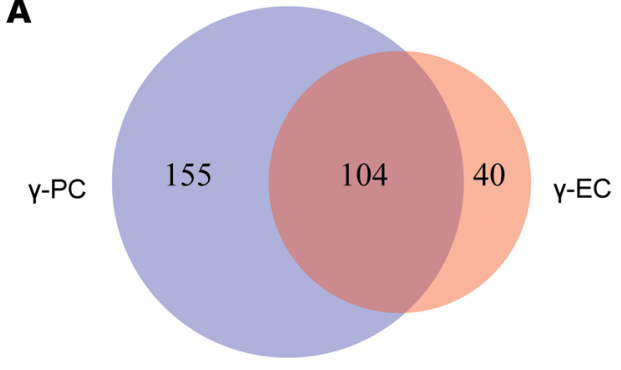

B

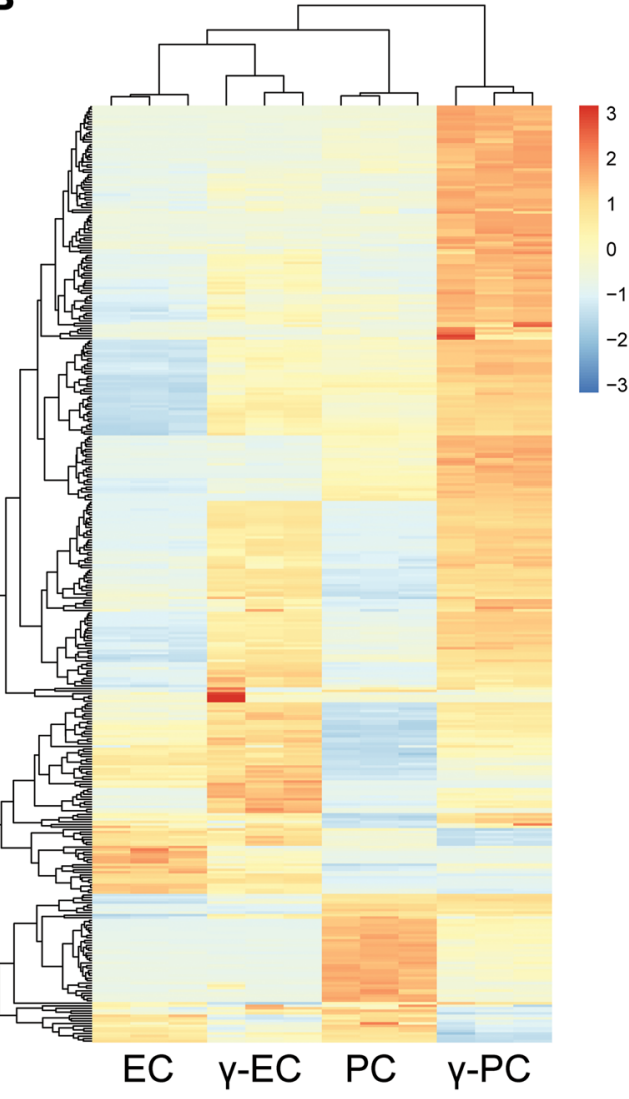

C

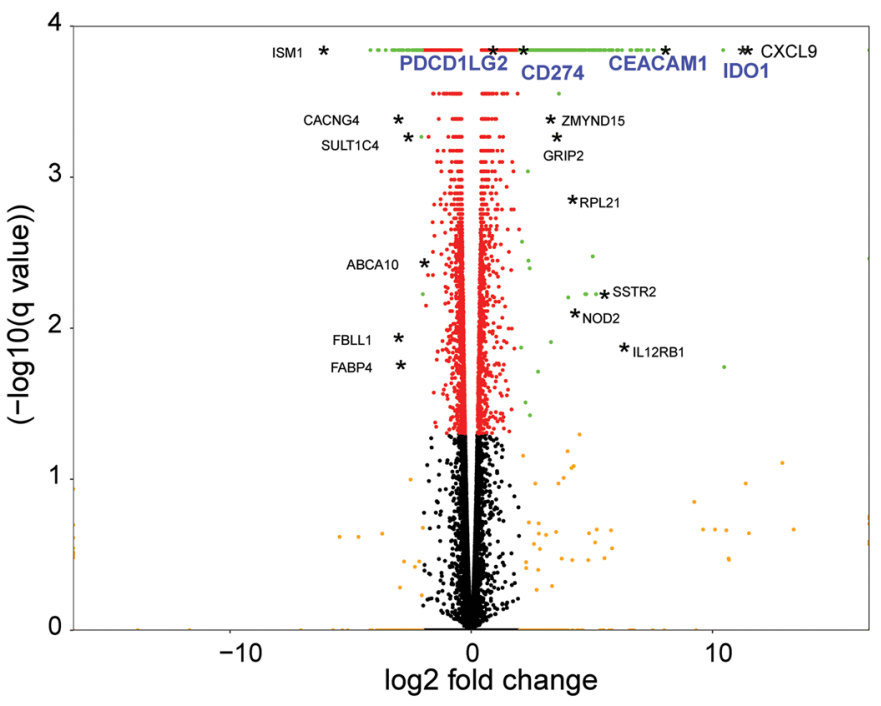

D

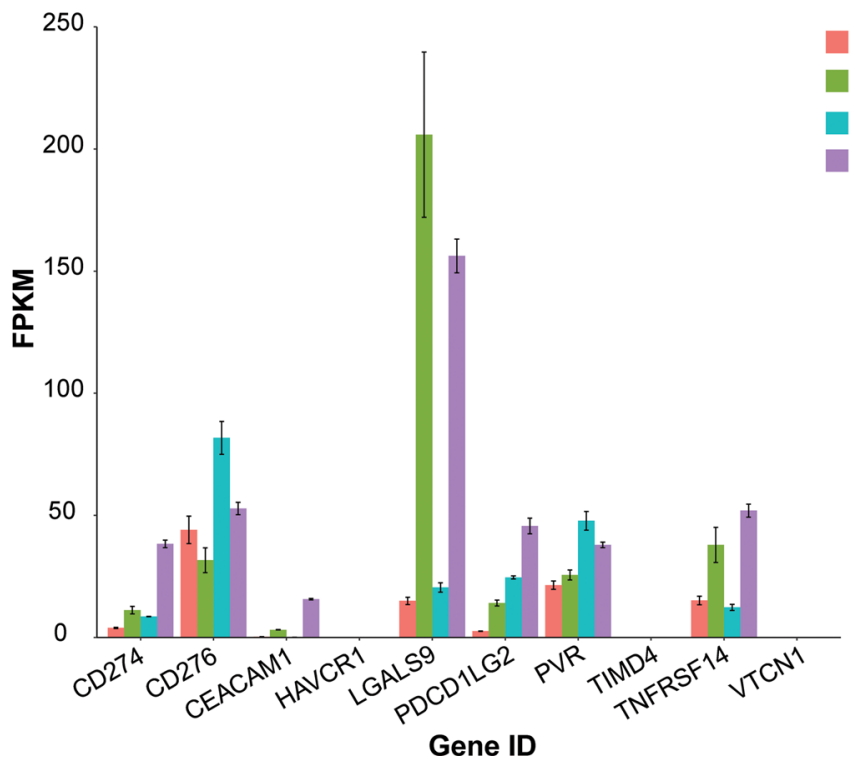

EC

$Y-E C$ $P C$ Y-PC

Figure 3. RNAseq analysis comparing transcriptome signatures of ECs, $\gamma$-ECs, PCs, and $\gamma$-PCs revealed candidate inhibitory molecules expressed by $\gamma$-PCs. (A) Venn diagram of the significantly upregulated genes with a $\log _{2}$ (fold change) $\left(\log _{2} \mathrm{FC}\right)>2$ after stimulation with IFN- $\gamma(50 \mathrm{ng} / \mathrm{ml}$ ) for 48 hours in endothelial cell $(\gamma-E C)$ and pericyte $(\gamma-P C)$ transcriptomes. (B) Heatmap of the relative gene expression levels (fragments per kilobase of transcript per million mapped reads, FPKM) for each gene (row) and each sample (column). Only genes significantly differentially expressed with a log 2 FC $>2$ after stimulation in both ECs and PCs are shown $(n=372)$. Rows and columns are hierarchically clustered. Expression values are scaled by row. (C) Volcano plot comparing FDR-adjusted $P$ value (- $\log _{10}\left[q\right.$ value]) as a function of $\log _{2}$ FC between $\gamma$-PC and unstimulated PC samples. Red dots are genes with a $q$ value $<0.05$; orange dots are genes with a $\log _{2} \mathrm{FC}>2$; and green dots are genes significantly differentially expressed with a log $2 \mathrm{FC}>2$. Black asterisks highlight examples of gene names belonging to the green dot category ( $q$ value $<0.05$ and $\log _{2} F C>2$ ). Specifically, gene names highlighted in blue (PDCD1LC2, CD274, CEACAM1, and ID01) are genes related to T cell activation and proliferation, and were selected for further experimentation. (D) Gene expression profile of negative costimulatory molecules, including PD-L1 (CD274), B7-H3 (CD276), CEACAM1, TIM-1 (HAVCR1), galectin-9 (LCALS9), PD-L2 (PDCD1LG2), CD155 (PVR), TIM-4 (TIMD4), HVEM (TNFRSF14), and B7-H4 (VTCN1) in ECs, $\gamma$-ECs, PCs, and $\gamma$-PCs.

IL-2, $\gamma$-PCs potently suppressed CD8 ${ }^{+} \mathrm{T}_{\mathrm{EM}}$ proliferation despite expressing higher levels of MHC class I molecules (Figure $2 B$ ), similar to their effects on $C D 4^{+} T_{E M}$.

The growth-stimulating effects of IL-2, indispensable in vitro, can be replaced in vivo by provision of other cytokines, derived from various different tissue-resident cell types. These other cytokines, like IL-2, signal through receptors that utilize $\gamma_{c}$ and associated JAK3 kinase. One such cytokine that is particularly relevant for activating $\mathrm{CD} 8^{+} \mathrm{T}$ cell function is IL-15. We therefore examined whether the observed inhibitory 
effects of $\gamma$-PCs on IL-2-induced CD8 ${ }^{+} \mathrm{T}_{\mathrm{EM}}$ proliferation may also extend to IL-15. Both unstimulated ECs and $\gamma$-ECs effectively induced $\mathrm{CD} 8^{+} \mathrm{T}_{\mathrm{EM}}$ proliferation that was enhanced by exogenous IL-15 (Supplemental Figure 2A). Unstimulated PCs induced $\mathrm{CD}^{+} \mathrm{T}_{\mathrm{EM}}$ proliferation that was also significantly enhanced by addition of IL-15 instead of IL-2. However, similar to their inhibition of IL-2-induced proliferation, $\gamma$-PCs potently suppressed IL-15-induced $\mathrm{CD} 8^{+} \mathrm{T}_{\mathrm{EM}}$ proliferation (Supplemental Figure $2 \mathrm{~B}$ ).

IL-2 and IL-15 both produce a common early signaling response in T cells bearing the relevant receptor, namely JAK3-mediated phosphorylation of tyrosine residues in STAT5. STAT phosphorylation can be blocked by the presence of various members of the silencer of cytokine signaling (SOCS) protein family (16). Proximal receptor function, or its inhibition, can thus be assessed by the induction or absence of rapid increases in STAT5 phosphorylation as assessed by flow cytometry. To examine whether the inhibition of allogeneic $\mathrm{T}_{\mathrm{EM}}$ proliferation by $\gamma$-PCs is a result of unresponsiveness to cytokine signaling, we examined the phosphorylation status of STAT5 in $\mathrm{T}_{\mathrm{EM}}$ cocultured with PCs or $\gamma$-PCs. Despite its failure to trigger T cell proliferation, IL-2 induced similar increases in STAT5 phosphorylation in CD8 ${ }^{+} \mathrm{T}_{\mathrm{EM}}$ that have interacted with either untreated PCs or $\gamma$-PCs (Supplemental Figure 3A). Similarly, IL-15 also readily induced phosphorylation in $\mathrm{CD}^{+} \mathrm{T}_{\mathrm{EM}}$ cocultured with either PCs or $\gamma$-PCs (Supplemental Figure 3B), indicating that $\mathrm{T}_{\mathrm{EM}}$ remain responsive to cytokine stimulation despite proliferation arrest. Cumulatively, these data suggest that the inhibitory effects on $\mathrm{T}_{\mathrm{EM}}$ cell proliferation exerted by $\gamma$-PCs involve a downstream block in the cell cycle rather than inhibition of proximal events such as TCR signaling, cytokine production, or cytokine signaling.

IFN- $\gamma$ induces expression of several inhibitory molecules in PCs to a greater extent than in ECs. To identify candidate signals provided by $\gamma$-PCs that mediate inhibition of $\mathrm{T}_{\mathrm{EM}}$ proliferation, we performed RNA sequencing (RNAseq) analyses of ECs, $\gamma$-ECs, PCs, and $\gamma$-PCs. Differential gene expression analysis of $\gamma$-ECs and $\gamma$-PCs against the nonactivated respective cell type revealed that while IFN- $\gamma$ upregulates 104 genes in both ECs and PCs, 40 gene transcripts are specifically upregulated in ECs only, and 155 genes are upregulated in PCs only $\left(\log _{2}\right.$ [fold change] $>2$ ) (Figure 3A). Hierarchical clustering of transcriptome signatures of ECs, $\gamma$-ECs, PCs, and $\gamma$-PCs highlights the dynamic effects of IFN- $\gamma$ signaling that differ remarkably at the transcription level in ECs versus PCs (Figure 3B). Two gene sets pertaining to regulation of $\mathrm{T}$ cell proliferation (Positive Regulation of $\mathrm{T}$ cell Proliferation and Negative Regulation of T cell Proliferation) were selected for gene set enrichment analysis (GSEA). $\gamma$-ECs trended towards enrichment of gene set Positive Regulation of T cell Proliferation, whereas $\gamma$-PCs showed robust enrichment of gene set Negative Regulation of T cell Proliferation (FDR $=0.10)$ (Supplemental Figure 4A), consistent with our experimental findings that IFN- $\gamma$ has opposite effects on regulating allogeneic $\mathrm{T}_{\mathrm{EM}}$ proliferation in response to ECs and PCs.

Bioinformatic analyses of RNAseq identified specific candidate IFN- $\gamma$-induced genes that were more strongly induced in PCs than ECs and that have been linked to immunoregulation. IDO1 was one of the most highly upregulated gene transcripts in $\gamma$-PCs, and $\gamma$-PCs expressed a much higher level of IDO1 transcripts than did $\gamma$-ECs (Figure 3C and Supplemental Figure 4B). In addition, IFN- $\gamma$ also induced PCs to upregulate programmed death-ligand 1 (PD-L1) and PD-L2, and the induced expression level was higher than that expressed by $\gamma$-ECs at both the mRNA level (Figure 3, C and D) and at the level of surface expression (Supplemental Figure 5A). mRNA encoding carcinoembryonic-antigen-related cell-adhesion molecule 1 (CEACAM1), a cell surface protein that negatively regulates $\mathrm{T}$ cell functions through homophilic binding to CEACAM1 or by heterophilic binding to T cell immunoglobulin domain and mucin domain-3 (TIM-3) receptors, both of which are expressed on $\mathrm{T}_{\mathrm{EM}}$ (17), was induced in both ECs and PCs by IFN- $\gamma$ stimulation, although $\gamma$-PCs expressed a higher level of CEACAM1 transcripts than $\gamma$-ECs (Figure 3D). Assessing surface expression of CEACAM1 using 3 different CEACAM1 antibodies by flow cytometry yielded inconsistent results, possibly due to sensitivity of the relevant epitope to enzymes used for cell suspension. However, both cell types appeared to express similar levels of CEACAM1 protein assessed by Western blot, and the bands were of equal molecular weight, suggesting that both cell types expressed the same CEACAM1 isoform (Supplemental Figure 5B). Of note, galectin 9, an alternative ligand to TIM-3 receptors, was highly upregulated in both $\gamma$-ECs and $\gamma$-PCs, but, in this case, the level expressed by $\gamma$-ECs was slightly higher than in $\gamma$-PCs (Figure 3D). mRNAs encoding other negative costimulatory molecules, including HVEM, B7-H3, B7-H4, VISTA, and CD155, were not upregulated by IFN- $\gamma$ in either ECs or PCs (Figure 3D). Protein expression levels of these negative costimulatory molecules were also unchanged as assessed by flow cytometry (Supplemental Figure 5A). 
A

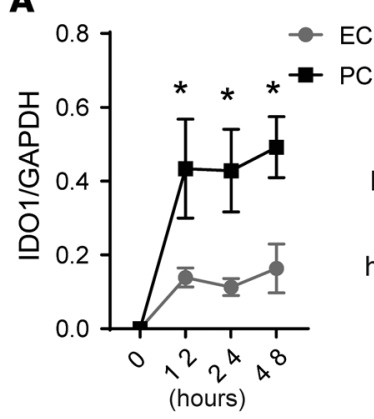

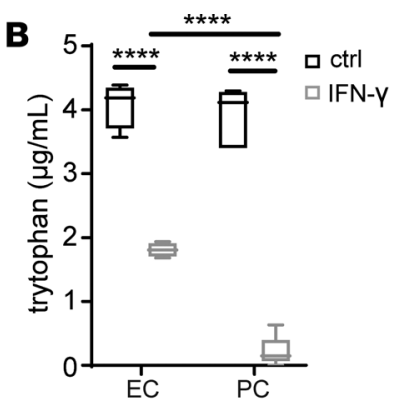

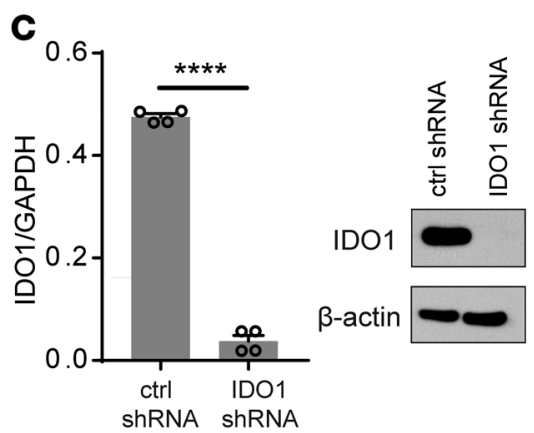

$$
\text { D }
$$

CD4
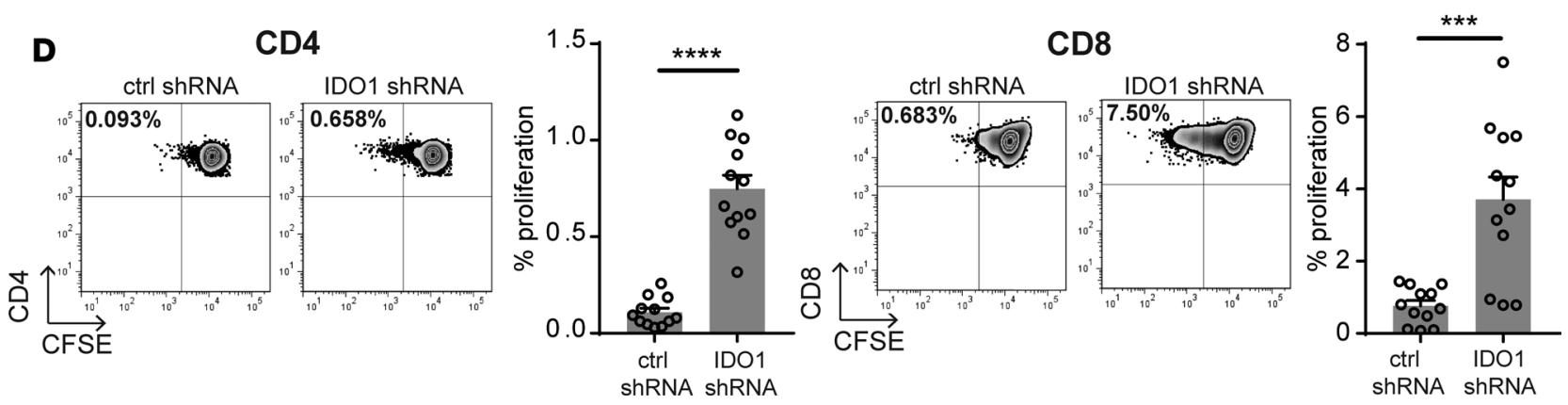

Figure 4. ID01 inhibition ameliorates $\gamma$-PC-mediated suppression of $T_{E M}$ proliferation. (A) qRT-PCR and Western blot analysis of IDO1 levels expressed by unstimulated endothelial cells (ECs), IFN- $\gamma$-activated ECs ( $\gamma$-ECs), unstimulated pericytes (PCs), or IFN- $\gamma$-activated PCs $(\gamma$-PCs) ( $n=4$, two-way ANOVA, SEM). (B) Measurement of tryptophan concentration in conditioned media of ECs, $\gamma$-ECs, PCs, or $\gamma$-PCs $(n=4$, SEM). (C) IDO1 expression by IFN- $\gamma$-stimulated control and ID01-knockdown PCs assessed by qRT-PCR or immunoblotting (representative data demonstrating the degree of IDO knockdown achieved in 2 independent PC donors is shown). (D) CD4+ (left) or CD8 (right) $\mathrm{T}_{E M}$ proliferation after coculture with IFN- $\gamma$-stimulated control or IDO1-knockdown PCs. Recombinant IL-2 $(25 \mathrm{U} / \mathrm{ml})$ was added on day 3 of coculture. FACS plots show a representative experiment using CSFE dye dilution to assess proliferation $\left(n=12, t\right.$ test, SEM). ${ }^{*} P<0.05,{ }^{* * *} P<0.001,{ }^{* * *} P<0.0001$.

Inhibition of IDO1 in $\gamma$-PCs reduces suppression of $T_{E M}$ proliferation. IDO1 is thought to inhibit $\mathrm{T}$ cell proliferation by degrading free tryptophan, which $\mathrm{T}$ cells must acquire from their environment in order to synthesize new proteins. To link the increased levels of mRNA encoding IDO1 to functional suppression of T cells, we first verified that IFN- $\gamma$ upregulated a significantly higher level of IDO1 transcript and protein in human PCs than in human ECs by quantitative real-time reverse transcription PCR (qRT-PCR) and immunoblotting (Figure 4A). Consistent with these determinations of IDO1 expression level, $\gamma$-PCs depleted extracellular tryptophan at a significantly faster rate than did $\gamma$-ECs (Figure 4B). Chemical inhibition of IDO1, which we had employed in the past in assays using total $\mathrm{CD}^{+} \mathrm{T}$ cells, was only minimally effective at restoring $\mathrm{T}_{\mathrm{EM}}$ proliferation (9). To more definitively examine the role of IDO1 in $\gamma$-PC-induced inhibition of $\mathrm{T}$ cell proliferation, we knocked down IDO1 expression in PCs through shRNA transduction. The efficiency of knockdown was confirmed by comparing levels of both IDO1 mRNA and protein of $\gamma$-PCs transfected with control shRNA or shRNA targeting IDO1 (Figure 4C). Knockdown of IDO1 largely and consistently reversed $\gamma$-PC-mediated inhibition of proliferation of both $\mathrm{CD}^{+}$and $\mathrm{CD}^{+} \mathrm{T}_{\mathrm{EM}}$ when tested with multiple different donor pairs (Figure 4D).

$\gamma$-PC-expressed ligands for $T$ cell $P D-1$ or TIM-3 have minimal effects on inhibition of $T_{E M}$ proliferation. We next assessed whether IFN- $\gamma$-induced negative costimulatory molecules, such as PD-1 ligands and TIM-3 ligands may also contribute to the immunoregulatory functions of $\gamma$-PCs. Blocking monoclonal antibodies against PD-L1 plus PD-L2 or against TIM-3, the T cell surface receptor for both CEACAM1 and galectin 9, had no significant effect on $\mathrm{CD}^{+}$or $\mathrm{CD}^{+} \mathrm{T}_{\mathrm{EM}}$ cell proliferation to allogeneic $\gamma$-PCs, although blocking PD-L1 and PD-L2 had a small enhancing effect on proliferation to $\gamma$-ECs (Figure 5, A and B). There is no source available to us to obtain a blocking antibody against human CEACAM1 to test for homophilic inhibitory effects. Therefore, to explore this possibility we knocked down CEACAM1 in PCs with either of 2 different siRNA that each produced significant reduction in CEACAM-1 protein expression. One of the two consistently reduced the inhibitory effects of $\gamma$-PCs, but the other did not, and a third siRNA that produced only partial silencing of CEACAM1 produced inconsistent effects on $\mathrm{T}_{\mathrm{EM}}$ responses (data not shown). We view these experiments on the role of CEACAM1 as being inconclusive. 
A

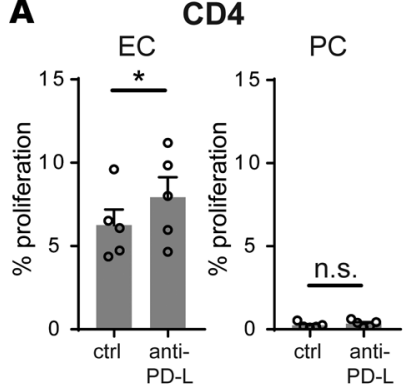

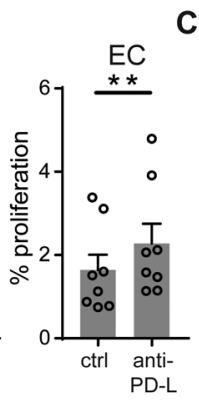

CD8

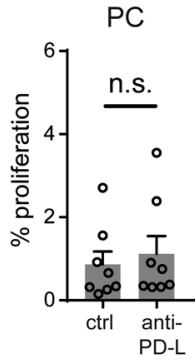

CD8

B

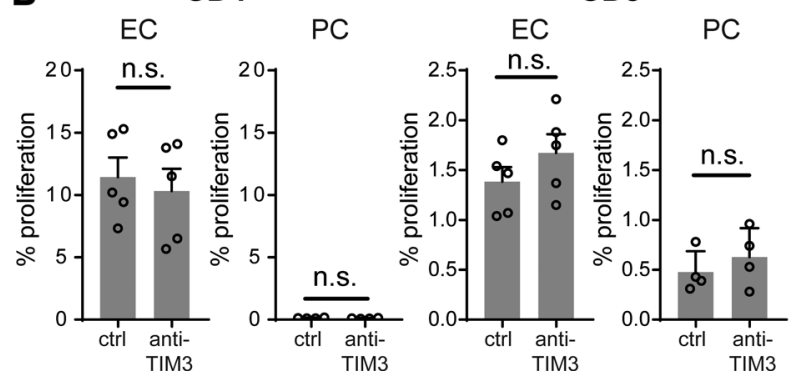

Figure 5. $\gamma$-PC-expressed PD-1 or TIM-3 ligands have minimal effects on inhibition of $T_{E M}$ proliferation. (A) Proliferation of allogeneic $T_{E M}$ after coculture with IFN- $\gamma$-pretreated endothelial cells ( $\gamma$-ECs) or pericytes ( $\gamma$-PCs) in the presence of anti-human PD-L1 plus anti-human PD-L2 blocking antibodies or isotype control $(10 \mu \mathrm{g} / \mathrm{ml}$ for each antibody). Recombinant IL-2 $(25 \mathrm{U} / \mathrm{ml})$ was added on day 3 of coculture, and CFSE dilution was assessed on day 7 ( $n=5-8$, $t$ test, SEM). (B) Proliferation of allogeneic $T_{E M}$ after coculture with $\gamma$-ECs or $\gamma$-PCs in the presence of anti-human TIM-3 blocking antibodies $(10 \mu \mathrm{g} / \mathrm{ml}$ for each antibody) or isotype control. Recombinant IL-2 $(25 \mathrm{U} / \mathrm{ml})$ was added on day 3 of coculture, and proliferation was assessed by CFSE dilution on day 7 ( $n=$ 4-5, $t$ test, SEM). ${ }^{*} P<0.05,{ }^{* *} P<0.01$. n.s., not significant.

Human PCs express IDO1 and CEACAM1 in vivo in a skin allograft rejection humanized mouse model and in biopsy samples of acutely rejected renal allografts. Just as HUVECs have been widely used to produce generalizable findings regarding EC immune functions, we have used human placental PCs to obtain generalized findings about PC immune functions. However, similar to ECs, PCs from different sites are likely to be heterogeneous and, more importantly, culturing of human PCs may change their phenotype and functions. It is difficult for practical and ethical reasons to conduct an investigation of human PC function in vivo, and anatomic heterogeneity cannot be adequately assessed by comparing cells cultured from different sites. To determine if the effects of IFN- $\gamma$ on PC expression of inhibitory molecules that we have seen in culture actually occur in human allograft rejection, we used a model of T cell-mediated rejection of human skin implanted on immunodeficient mouse hosts (18). Human dermal microvessels, formed from human ECs and PCs, are retained and perfused in these grafts, and these vessels are typically destroyed by alloreactive $\mathrm{T}_{\mathrm{EM}}$ approximately 2 weeks after adoptive transfer of human peripheral blood mononuclear cells (PBMCs) allogeneic to the skin donor. At earlier times, the human vessels respond to human cytokines, including IFN- $\gamma$, produced by the transferred human T cells. To study PC responses in situ, we harvested the skin grafts on day 7 , a time at which there is early but limited infiltration of $\mathrm{T}$ cells into skin tissues and prior to significant damage of the graft (Supplemental Figure 6). We observed that dermal microvascular PCs in vessels surrounded by sparse infiltrates of allogeneic human T cells were induced to express IDO1 and CEACAM1 (Figure 6, A-D). As in our cell culture experiments, human dermal microvascular ECs also express IDO1, but PCs were induced to express significantly higher levels of CEACAM1 than ECs (Figure 6, B and D).

In a final series of experiments, we examined whether PCs express IDO1 and CEACAM1 in biopsies from human renal allografts exhibiting acute $\mathrm{T}$ cell-mediated rejection. Similar to observations made in our humanized mouse model of skin allograft rejection, PCs in renal allografts undergoing acute rejection also upregulated IDO1 and CEACAM1 in comparison with PCs in renal allografts in control biopsies taken at the time of transplantation (time zero) not showing rejection (Figure 7, A-D). Microvascular expression of IDO1 and CEACAM1 was largely restricted to PCs and not displayed by the ECs lining the same vessel segments (Figure 7, B and D). These observations suggest that IFN- $\gamma-$ mediated responses of cultured human placental PCs also occur in human renal microvascular PCs of allografts during early stages of cell-mediated rejection. Although the numbers of such vessels are too few to report as a finding with certainty, the occasional arteriole in the same biopsies also showed similar localization of IDO1 and CEACAM1 to multilayered vascular smooth muscle cells and not ECs, suggesting this response may be shared by both mural vascular cell types. 
A
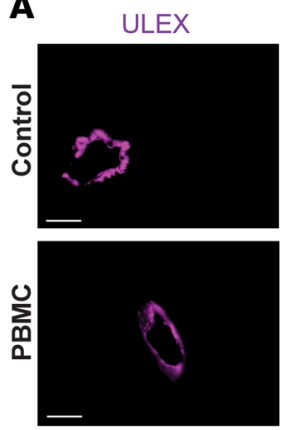

C
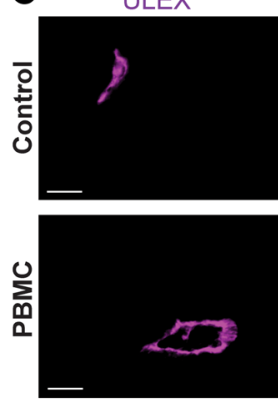

a-SMA
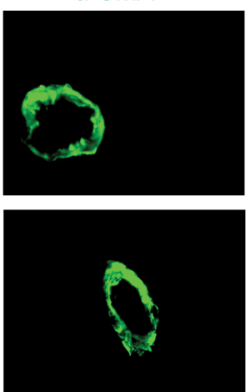

a-SMA
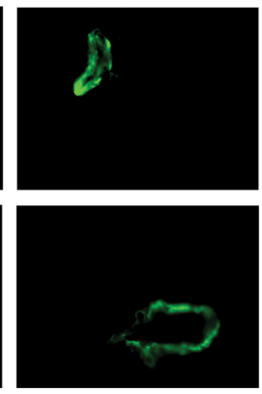
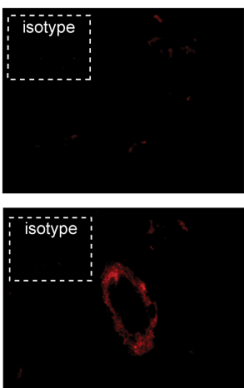

CEACAM1
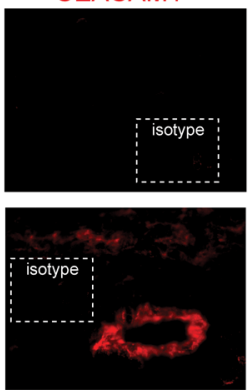

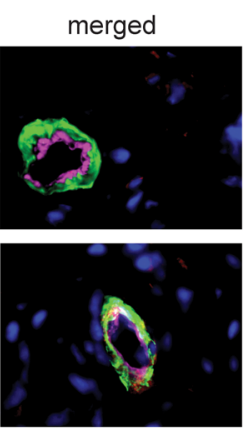

merged
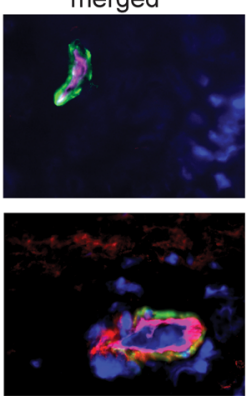

B

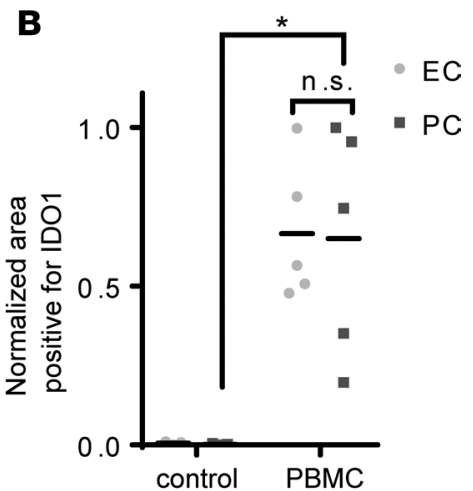

D

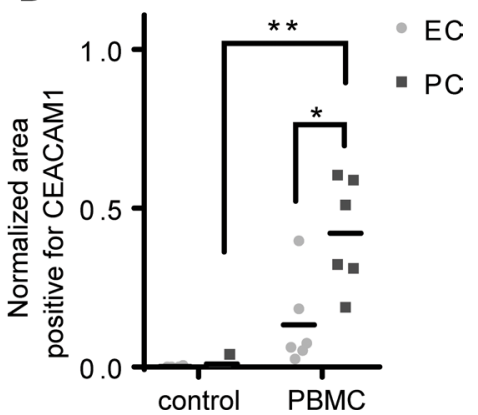

Figure 6. Dermal PCs express ID01 and CEACAM1 in vivo in a skin allograft rejection humanized mouse model. Immunofluorescence analysis of human skin allograft implanted on immunodeficient mice receiving control saline or human PBMCs. Skin grafts were harvested on day 7 after injection. (A) Freshly frozen tissues were co-stained for Ulex europaeus agglutinin I (ULEX), $\alpha$-smooth muscle actin ( $\alpha$-SMA), and IDO1. Images shown are representative of 3 skin grafts. (B) Quantification of IDO1 colocalization to endothelial cells (ECs) or pericytes (PCs) on ULEX+ $\alpha$-SMA ${ }^{+}$microvessels ( $t$ test, mean). (C) Tissues were costained for ULEX, $\alpha$-SMA, and CEACAM1. Results shown are representative of 3 skin grafts. (D) Quantification of CEACAM1 colocalization to ECs or $\mathrm{PC}$ s on ULEX+ $\alpha-S M A^{+}$microvessels ( $t$ test, mean). Scale bars: $16 \mu \mathrm{m}$. ${ }^{*} P<0.05,{ }^{* *} P<0.01$. n.s., not significant.

\section{Discussion}

Acute cell-mediated allograft rejection is initiated by recruitment of circulating host $\mathrm{T}$ cells, and in humans, is mediated at early times by circulating alloreactive $\mathrm{T}_{\mathrm{EM}}$ already present in the circulation, with no need for priming in secondary lymphoid organs. Typically, $\mathrm{T}_{\mathrm{EM}}$ exit the blood through postcapillary venules and the process of extravasation involves close sequential interactions with both of the 2 principal microvascular cells that form the venular wall, namely ECs and PCs. Much is known about the interactions of human ECs with $\mathrm{T}_{\mathrm{EM}}$ but very little is known about the immunological functions of human PCs. In this study, we used cultured human placental microvascular PCs, to analyze the immunomodulatory effects of PCs on allogeneic peripheral blood $\mathrm{T}_{\mathrm{EM}}$ cells and compared our findings with parallel cocultures using ECs cultured from the umbilical veins of the same donors. Our key findings are that resting PCs are capable of activating alloreactive $\mathrm{T}_{\mathrm{EM}}$ cells, but upon IFN- $\gamma$ stimulation, PCs instead suppress $\mathrm{T}$ cell expansion. Clearly, the immunoregulatory functions of PCs exposed to IFN- $\gamma$ can be overcome in vivo as rejection does occur, perhaps because some vascular beds show a very low ratio of PCs to ECs, but our findings raise the possibility that approaches to manipulate graft PCs, e.g., with antibody targeting of therapeutics, could be developed to complement other approaches to improve graft survival. This may be particularly useful in protecting tissue-engineered grafts in which genetically altered PCs can be introduced into microvessels (19). It is also worth considering that tumors may alter microvascular PCs in a manner that suppresses immune responses and our new findings with human PCs may conversely be applied to enhance antitumor immunity (8). Interestingly, IFN- $\gamma$ has different effects on ECs and PCs, enhancing the capacity of ECs to activate alloreactive $\mathrm{T}$ cells while inducing inhibitory signals on PCs that suppress $\mathrm{T}$ cell proliferation. The inhibitory effect targets $\mathrm{T}$ cell proliferation induced by growth-promoting cytokines that bind to receptors using the $\gamma_{\mathrm{c}}$ signaling subunit, but not $\mathrm{T}$ cell cytokine production nor proximal signaling events such as STAT5 phosphorylation. Further, based on evaluating candidates from an RNAseq analysis, we identified PC expression of IDO1 as the principal mechanism accounting for negative regulation of $\mathrm{T}$ cell proliferation by $\gamma$-PCs. CEACAM1 may also be a relevant inhibitory molecule, although our conclusions in this 
A
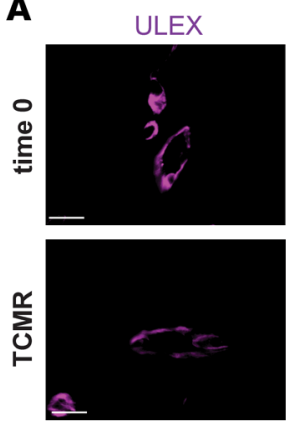

C
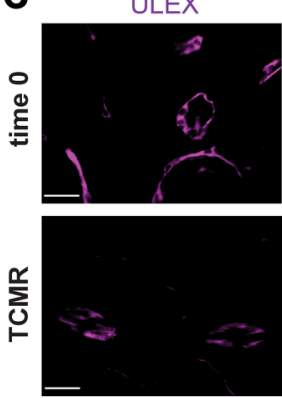

a-SMA
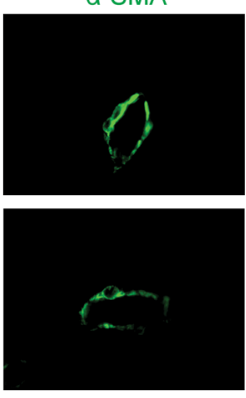

a-SMA
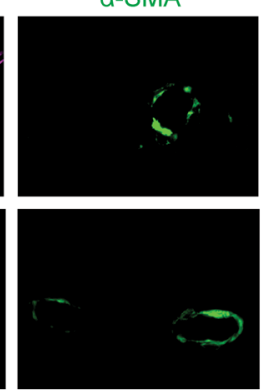
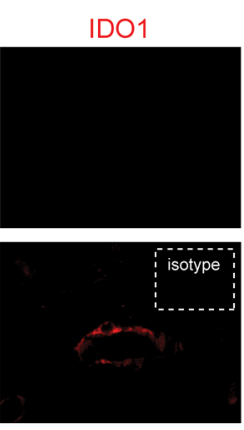

CEACAM1

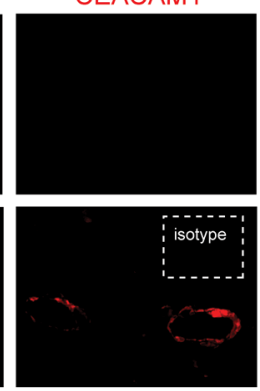

merged

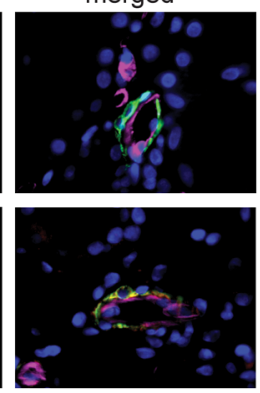

merged
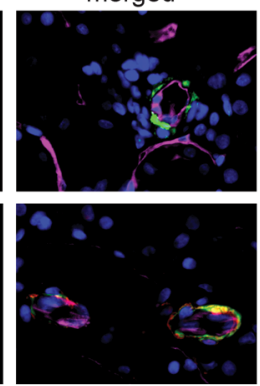

B
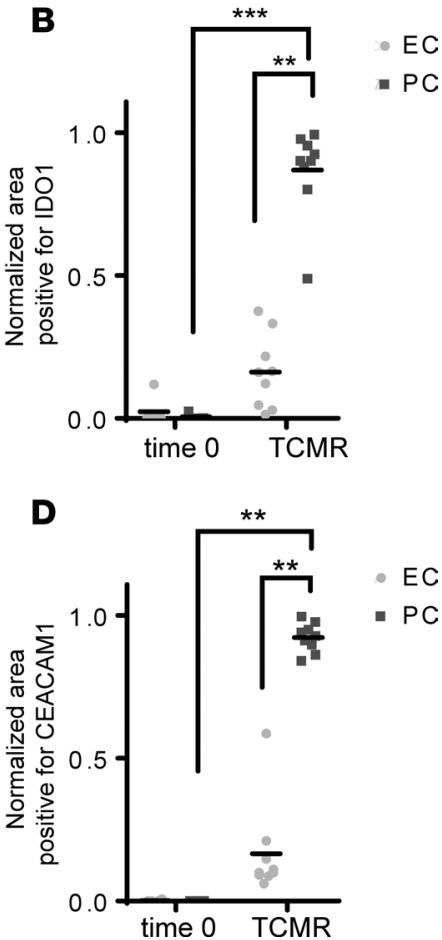

Figure 7. PCs express IDO1 and CEACAM1 in acutely rejected renal allografts. Immunofluorescence analysis of control biopsy samples taken at the time of transplant (time 0 ) and renal allografts undergoing T cell-mediated rejection (TCMR). (A) Tissues were costained for Ulex europaeus agglutinin I (ULEX), $\alpha$-smooth muscle actin ( $\alpha$-SMA), and IDO1. (B) Quantification of ID01 colocalization to endothelial cells (ECs) or pericytes (PCs) on ULEX+ $\alpha-S M A^{+}$microvessels ( $t$ test, mean). (C) Tissues were costained for ULEX, $\alpha$-SMA, and CEACAM1. (D) Quantification of CEACAM1 colocalization to ECs or PCs on ULEX ${ }^{+} \alpha-S M A^{+}$microvessels ( $t$ test, mean). Results shown are representative of 4 control biopsy samples and 4 renal allografts undergoing TCMR. Scale bars: $16 \mu \mathrm{m}$. ${ }^{* *} P<0.01,{ }^{* *} P<0.001$.

case are equivocal. While our in vitro studies rely heavily on human placental PCs, a prototypical population similar to the use of HUVECs to study ECs, we also find that PC expression of IDO1 and CEACAM1 can be observed in human skin during early stages of rejection by allogeneic $\mathrm{T}_{\mathrm{EM}}$ as well as in human renal biopsies showing evidence of $\mathrm{T}$ cell-mediated rejection. While the response of PCs appears to be consistent, the response of ECs appears somewhat more variable.

Whereas the involvement of microvascular ECs in the inflammatory response has been thoroughly established, an active role of PCs in this process has only been much more recently appreciated $(20,21)$. The anatomical localization of microvascular PCs, in juxtaposition to the endothelium that lines the vascular lumen, is situated at a unique and pivotal position to influence extravasating and newly extravasated leukocytes and their interstitial activities. Recent studies have identified essential roles of PCs in guiding neutrophil extravasation and subsequent guidance in early interstitial migration following tissue entry (7, 22). We previously reported that PCs may be more responsive to specific cytokines than ECs. For example, PCs are the primary cell type responsive to IL-17 in the microvasculature and IL-17-activated PCs, but not ECs, can promote neutrophil effector cytokine production, phagocytosis, and survival (23). Much less is known about how PCs influence T cell recruitment or activation. We previously demonstrated that $\gamma$-PCs did not induce cytokine production or proliferation of allogeneic peripheral blood CD $4^{+} \mathrm{T}$ cells (consisting of both naive and memory subsets), and instead induced T cell anergy (9). The experiments reported here advance these earlier studies in 3 ways. First, because measurable activation of alloreactive human CD4 ${ }^{+}$ $\mathrm{T}$ cells isolated from blood requires direct recognition of non-self-allelic forms of class II MHC molecules and because cultured human vascular cells do not constitutively express class II MHC molecules, these older experiments used treatment of the PCs (and ECs) with IFN- $\gamma$ to induce expression of class II MHC molecules. In the current study, we compared retroviral transduction to overexpress CIITA with IFN- $\gamma$ pretreatment, allowing us to separate the much more specific effects of the former approach on class II MHC expression from the more global immunomodulatory effects of the latter. Second, given the pivotal role of the $\mathrm{T}_{\mathrm{EM}}$ subset in targeting graft microvessels to initiate early acute allograft rejection in humans, we specifically isolated these populations from the blood to avoid confounding effects of other $\mathrm{T}$ cell subsets. 
Since $\mathrm{T}_{\mathrm{EM}}$ typically constitute fewer than $10 \%$ of the total $\mathrm{T}$ cells in peripheral blood, it is easy to overlook or obscure their responses without using $\mathrm{T}_{\mathrm{EM}}$-enriched populations. This difference may account for why our previous study did show an effect on cytokine production as well as proliferation. Third, given the importance of $\mathrm{CD}^{+}$cytotoxic $\mathrm{T}$ cells as a primary mediator of allograft rejection, we examined for the first time to our knowledge the potential influence of PCs on alloreactive $\mathrm{CD} 8^{+} \mathrm{T}_{\mathrm{EM}}$, the population that gives rise to cytotoxic $\mathrm{T}$ lymphocytes. Our data on $\mathrm{PC}-\mathrm{T}_{\mathrm{EM}}$ interactions corroborate our previous finding in that $\mathrm{CD}^{+} \mathrm{T}_{\mathrm{EM}}$, similar to total $\mathrm{T}$ cells, do not proliferate in response to $\gamma$-PCs and we can now report that, in contrast to $\gamma$-ECs, $\gamma$-PCs also fail to activate $\mathrm{CD}^{+} \mathrm{T}_{\mathrm{EM}}$. The fundamental new observation is that neither resting PCs nor CIITA-PCs appear inhibitory and are actually fairly effective activators of alloreactive $\mathrm{T}_{\mathrm{EM}}$ so that immunoregulation arises from the effects of IFN- $\gamma$ on PCs. To sort out mechanisms, we utilized RNAseq to identify candidate gene products induced preferentially by IFN- $\gamma$ in PCs versus ECs. IDO1 as well as several known membrane-bound inhibitory ligands were all strongly upregulated on PCs by IFN- $\gamma$. Functional experiments singled out IDO1 as the principal inhibitory mechanism.

Of the few previously published reports on PC-T cell interactions in various contexts, PCs seemed to exhibit properties that are largely immunosuppressive in $\mathrm{T}$ cell activation $(8,9,24)$. Our data suggest that these immunoregulatory effects of PCs on T cell proliferation are induced by IFN- $\gamma$. Interestingly, mouse tumor vessel PCs, which like the human placental microvessel PCs used in our study have also been shown to inhibit $\mathrm{T}$ cell proliferation and induce anergy, also suppressed cytokine production by $\mathrm{CD} 4^{+} \mathrm{T}$ cells, something which we did not observe (8). In addition to the use of different sources of tissues, the inhibitory effect on cytokine production by mouse tumor vessel PCs was most obvious using antigen-primed, CD3/CD28-preactivated $\mathrm{T}$ blasts, whereas we study resting $\mathrm{T}_{\mathrm{EM}}$. Other differences, such as species (mouse vs. human) or the culture milieu, may also have contributed to the effects on cytokine production. Species differences are likely to be very important. Mesenchymal stromal cells (MSCs) share many common characteristics with PCs and their potent immunosuppressive role has been extensively characterized in disease models as well as in clinical trials. Direct comparison of MSCs from 7 different mammalian species revealed that MSCs from human, monkey, and pig use IDO1, but not inducible nitric oxide synthase (iNOS), to inhibit immune response, whereas MSCs from mouse, rat, rabbit, and hamster exert immunosuppression predominately through expression of iNOS instead of IDO1 (25). The strikingly distinct mechanisms underlying MSC-mediated immunosuppression among mammalian species highlights the importance of studying human PCs in understanding their potential role in mediating immune tolerance in human diseases. This difference may also explain the differences we noted between effects on cytokine production by human placental microvessel PCs versus mouse tumor vessel PCs.

Our study reveals that IFN- $\gamma$ has important differential effects on ECs and PCs. Hierarchical clustering of the gene expression signature of ECs, $\gamma$-ECs, PCs, and $\gamma$-PCs highlights the dynamic effects of activation by IFN- $\gamma$ signaling that differ greatly at the transcription level in ECs versus PCs. As noted above, we found that IDO1 expression by $\gamma$-PCs is an important contributor to the inhibition of $\mathrm{T}$ cell proliferation. IDO1 functions by depleting the milieu around the cell of tryptophan, an essential amino acid for $\mathrm{T}$ cell expansion. While IFN- $\gamma$ also induces IDO1 in ECs, the expression level is much lower than in PCs. IDO1 induction serves as an explanation for our previous observations that $\gamma$-PCs may inhibit CD4 ${ }^{+} \mathrm{T}$ cell proliferation across a semipermeable transwell membrane, which suggested the mechanism of inhibition likely involves contact-independent paracrine signals (9). We note that in our prior experiments, we had tested a pharmacological inhibitor of IDO1, namely 1-methyl tryptophan, and did not fully rescue T cells from $\gamma$-PC inhibition. In pilot experiments conducted for the current study, we also found only partial benefit using this agent. However, transduction with shRNAs targeting IDO1 was highly effective at relieving $\gamma$-PC-mediated $\mathrm{T}$ cell inhibition, leading us to conclude that the level of pharmacological inhibition is simply inadequate to suppress the level of enzyme expressed by $\gamma$-PCs. In vivo, PCs face the interstitial side of the microvessels, whereas ECs face the blood stream. Consequently, it is probably futile for IDO1 expressed by ECs to exert any real effect on extracellular tryptophan levels, as blood levels will be rapidly replenished by flow. Interstitial levels, in contrast, may turn over quite slowly, allowing PCs to exert a significant effect on the local microenvironment. Our observation that IDO1 is induced in human microvascular PCs in living skin and kidney as an early event in allorejection lends credence to this hypothesis. IDO1 expression in response to IFN- $\gamma$ and TNF stimulation in vitro has been described in human brain PCs, but the consequence of tryptophan depletion by brain PCs in the context of immune tolerance has not been examined. In addition to consumption of this essential amino acid required for $\mathrm{T}$ cell metabolism, downstream metabolites produced 
by IDO1-catalyzed tryptophan degradation also may affect T cell differentiation. Specifically, IDO1 converts tryptophan into kynurenine, which binds and activates the aryl hydrocarbon receptor (AhR), thereby promoting Treg differentiation (26). Characterization of the metabolic pathway of L-tryptophan degradation in IDO1-expressing, IFN- $\gamma$-treated human brain PCs demonstrates that these cells produce significant levels of L-tryptophan metabolites kynurenine and picolinic acid, but not kynurenic acid or quinolinic acid (27). Functionally, human brain and placental PCs, and also PCs derived from human pluripotent stem cells may induce de novo formation of suppressive Tregs through TGF- $\beta$ production and expression of PD-ligands (28). To our knowledge, whether IDO1 expression by human PCs is involved in Treg induction has not been examined. Mouse liver PCs, also known as hepatic stellate cells, however, have been shown to promote the suppressive function of natural Treg through hepatic stellate cell-expressed IDO1 and activation of the aryl hydrocarbon receptor (29).

While IFN- $\gamma$ is often categorized as a proinflammatory cytokine, a role in counterregulation of immune response has been increasingly recognized. Early evidence suggesting the paradoxical properties of IFN- $\gamma$ in inflammation were mostly derived from observations made in animal models of autoimmunity, where exacerbation of experimental autoimmune encephalomyelitis and collagen-induced arthritis were observed in IFN- $\gamma$ or IFN- $\gamma \mathrm{R}$-deficient mice $(30,31)$. Similarly, in the context of solid organ rejection, accelerated rejection and reduced survival of heart, liver, and renal allografts also occur in mice deficient in IFN- $\gamma$ signaling (32-35). The precise mechanism of the IFN- $\gamma$-mediated tolerance induction in allograft is still an area of active investigation, but evidence thus far indicates that the immunoregulatory effect of IFN- $\gamma$ is multifactorial. The severe tissue necrosis and hemorrhage observed in exacerbated graft rejection in mice lacking IFN- $\gamma$ signaling has been shown to be mediated by neutrophils, suggesting that IFN- $\gamma$ likely also plays a role in inhibiting immune function of other leukocyte subsets in addition to effects on T cells (36). For instance, IFN- $\gamma$ has been shown to negatively regulate production of several key neutrophil chemoattractants in mice, including KC (CXCL-1) and MCP-2 (CCL-8) (37), and therefore deletion of IFN- $\gamma$ may permit increased infiltration of neutrophils during early graft rejection. On the adaptive immunity arm, IFN- $\gamma$ has been shown to exert its immunoregulatory role through suppression of $\mathrm{T}$ cell differentiation, particularly $\mathrm{Th} 2$ and Th17, promotion of Treg function, and importantly, induction of multiple negative costimulatory molecules. Our RNAseq analysis revealed that $\gamma$-PCs upregulate several membrane-bound inhibitory ligands, namely PD-L1, PD-L2, CEACAM1, and galectin 9, the latter 2 being known ligands for TIM-3 receptors on T cells. However, blocking PD-ligands and TIM-3 receptor is not sufficient in releasing the $\gamma$-PC-mediated inhibition of $\mathrm{T}_{\mathrm{EM}}$ proliferation. As we have noted, in addition to engagement of TIM-3, CEACAM1 can also deliver inhibitory signals through homophilic interactions with CEACAM1 on T cells. Whether this pathway may play a role in PC-mediated negative regulation of $\mathrm{T}$ cell function remains to be determined, and results of our siRNA experiments were ambiguous.

The immunoregulatory role of PCs described in the current study is most likely to be relevant during acute $\mathrm{T}$ cell-mediated rejection. It has been suggested by others that PCs may participate in an important way in the process of chronic graft rejection. Specifically, PCs normally maintain intimate contact with ECs and, from this position, provide both contact-dependent and paracrine signals known to play a role in stabilizing microvessels. Disruption of contacts between PCs and ECs, due either to PC migration or PC loss, could contribute to pathological angiogenesis and/or to microvessel rarefaction. Clinical analyses of renal, liver, and lung allograft rejection have revealed that destruction of microvasculature precedes chronic rejection and renders transplanted organs more susceptible to graft failure (38-41). In addition to their role in vessel stabilization, PCs, along with fibroblasts and possibly ECs, are viewed as precursors of myofibroblasts, the source of excessive matrix production associated with organ fibrosis (42). However, a fibrogenic role of PCs in chronic rejection have largely been extrapolated from studies of PCs in various other inflammatory contexts, and there is little actually known with certainty about the origins of the myofibroblasts present in grafts displaying chronic rejection, e.g., by fate mapping. Nevertheless, this alternative function of microvascular PCs is likely to be important and preservation of PC-EC contact could represent a therapeutic target for reducing microvessel loss and fibrosis in the setting of late graft failure.

In summary, we have provided evidence that while human PCs in an uninflamed environment have capacity to present antigen and activate allogeneic T cells, IFN- $\gamma$ converts PCs into negative regulators of allogeneic $\mathrm{T}$ cell response. This process is predominately mediated through IFN- $\gamma$ induction of IDO1 expression by PCs. We showed that IFN- $\gamma$ has differential effects on ECs versus PCs, namely that IFN- $\gamma$ activates ECs to promote alloreactive T cell activation but instead induces inhibitory properties of PCs. Findings from this study highlight the relevant roles of PCs, an often-ignored cell type, in the context of 
inflammation, and offer important mechanistic insights into how $\gamma$-PCs may be manipulated to promote allograft survival and possibly tumor immunotherapy.

\section{Methods}

\section{Isolation of primary human cells}

HUVECs. ECs, isolated from deidentified umbilical cords following enzymatic digestion by collagenase, were serially cultured on $0.1 \%$ gelatin-coated tissue culture plates in M199 medium (Gibco) containing 20\% FBS, $2 \mathrm{mM}$ L-glutamine, penicillin (100 U/ml), and streptomycin $(100 \mu \mathrm{g} / \mathrm{ml})$ supplemented with Endothelial Cell Growth Supplement (BD Bioscience) and porcine intestinal heparin (100 $\mu \mathrm{g} / \mathrm{ml}$, SigmaAldrich) (43). Cells were used between the first and fourth passage, at which time they uniformly express CD31 and are free of contaminating CD $45^{+}$leukocytes assessed by flow cytometry.

Human placental PCs. Samples of deidentified human placenta (approximately $10 \mathrm{~cm} \times 10 \mathrm{~cm} \times 3 \mathrm{~cm}$ ), taken from the region near the insertion of the umbilical cord, were manually minced and then subjected to collagenase digestion on a shaker for 3 hours at $37^{\circ} \mathrm{C}$. Microvessel segments were collected by sieving and PCs, obtained by explant outgrowth, were serially cultured in M199 medium (Gibco) containing 20\% FBS, $2 \mathrm{mM}$ L-glutamine, penicillin $(100 \mathrm{U} / \mathrm{ml})$, and streptomycin $(100 \mu \mathrm{g} / \mathrm{ml})(19)$. Cells were used between the second and sixth passage, at which time they expressed NG2, Thy-1 (CD90), and PDGFR $\beta$ as assessed by flow cytometry. Where indicated, ECs and PCs were isolated from the same donor.

Human PBMCS. Leukapheresis products were collected from deidentified healthy volunteer adult donors and mononuclear cells were further enriched by density gradient centrifugation using Lymphocyte Separation Medium (MP Biomedicals) per the manufacturer's guideline. Enriched PBMCs were aliquoted and then cryopreserved in 10\% DMSO/90\% FBS as previously described (14). Viability of subsequently thawed PBMCs was greater than $95 \%$ and alloreactivity was also preserved.

\section{Flow cytometric analysis of vascular cells}

ECs and PCs were treated with $50 \mathrm{ng} / \mathrm{ml}$ IFN- $\gamma$ (Gibco) or vehicle control for 72 hours. Cells were then trypsinized, washed with FACS staining buffer (1\% BSA in PBS), and subsequently stained with fluorophore-conjugated anti-human HLA-A,B,C (Biolegend, clone W6/32), HLA-DR (Biolegend, clone L243), PD-L1 (BD, clone MIH1), PD-L2 (BD, clone MIH18), HVEM (Biolegend, clone 122), B7-H3 (Biolegend, clone MIH42), B7-H4 (Biolegend, clone MIH43), CD155 (eBioscience, clone 2H7CD155), VISTA (R\&D Systems, clone 730804), or the appropriate isotype control antibodies for 30 minutes at $4^{\circ} \mathrm{C}$ before acquisition on an LSR II flow cytometer (BD).

\section{Isolation of $\mathrm{CD}^{+}$and $\mathrm{CD} 8^{+} \mathrm{T}_{\mathrm{EM}}$ from PBMCs}

Total $\mathrm{CD}^{+}$or $\mathrm{CD}^{+} \mathrm{T}$ lymphocytes were purified from PBMCs using Dynabeads CD4 or CD8 Positive Isolation Kit (Invitrogen), respectively, per the manufacturer's protocol. To obtain the resting $\mathrm{T}_{\mathrm{EM}}$ subset, naive $\mathrm{T}$ cells, central memory $\mathrm{T}$ cells, and activated $\mathrm{T}_{\mathrm{EM}}$ were depleted from the purified total $\mathrm{CD}^{+}$ or $\mathrm{CD}^{+}$populations using antibodies against human CD45RA (eBioscience, HI100), CCR7 (Biolegend, clone G043H7), CD62L (eBiosicence, clone DREG56), and HLA-DR (clone LB3.1), followed by removal of antibody-bound cells by magnetic separation using Dynabeads Pan Mouse IgG (Invitrogen). The isolated populations were routinely greater than $95 \% \mathrm{CD}^{2} 5 \mathrm{RA}^{-} \mathrm{CCR} 7^{-} \mathrm{CD} 62 \mathrm{~L}^{-} \mathrm{HLA}-\mathrm{DR}{ }^{-} \mathrm{CD} 45 \mathrm{RO}^{+} \mathrm{CD}^{+}{ }^{+}$or CD45RA-CCR7-CD62L-HLA-DR-CD45RO ${ }^{+} \mathrm{CD}^{-}{ }^{+} \mathrm{T}_{\mathrm{EM}}$ lymphocytes, respectively. $\mathrm{T}_{\mathrm{EM}}$ were then cultured with allogeneic vascular cell populations in RPMI 1640 media (Gibco) with 10\% FBS, 2\% L-glutamine, penicillin $(100 \mathrm{U} / \mathrm{ml})$, and streptomycin $(100 \mu \mathrm{g} / \mathrm{ml})$.

\section{Assay of $\mathrm{T}_{\mathrm{EM}}$ proliferation}

ECs or PCs were cultured in flat-bottom 48 -well plates for 72 hours with or without $50 \mathrm{ng} / \mathrm{ml} \mathrm{IFN- \gamma}$ (Gibco), at which time each contained approximately $1 \times 10^{5}$ vascular cells. $\mathrm{CD}^{+}$or $\mathrm{CD}^{+} \mathrm{T}_{\mathrm{EM}}$ were labeled with carboxyfluorescein succinimidyl ester (CFSE, $0.5 \mu \mathrm{M}$ for 15 minutes at room temperature) using the Cell Trace CFSE Cell Proliferation Kit (Invitrogen). CFSE-labeled CD4 ${ }^{+}$or CD8 ${ }^{+} \mathrm{T}_{\mathrm{EM}}(500,000$ cells) were then added to each well of the 48-well plate containing confluent unstimulated or IFN- $\gamma$-treated vascular cells (day 0). Recombinant human IL-2 (25 U/ml; eBioscience), $10 \mathrm{ng} / \mathrm{ml}$ recombinant human IL-15 (R\&D Systems), or vehicle control was added on day 3 of coculture where indicated. T cells were harvested 
on day 7, stained for CD4 or CD8 using conjugated primary antibodies (eBioscience), and assessed for CFSE dilution, an indicator of proliferation, on a BD LSRII Flow Cytometer using the BD FACSDiva Software. Where indicated, shRNA IDO1-transfected or CEACAM1 siRNA-transfected PCs were used as the vascular cell populations for coculture with $\mathrm{T}_{\mathrm{EM}}$. For experiments to identify inhibitory ligands on ECs or PCs, blocking antibodies targeting human PD-L1 (Biolegend, clone 29E.2A3) and PD-L2 (Biolegend, clone MIH18) or TIM-3 (Biolegend, clone F38-2E2), along with respective isotype controls, were added to the cocultures at day 0 at $20 \mu \mathrm{g} / \mathrm{ml}$ for each antibody.

\section{Assessment of STAT5 phosphorylation}

Cocultures of $\mathrm{CD}^{+} \mathrm{T}_{\mathrm{EM}}$ with unstimulated PCs or $\gamma$-PCs were set up as described above. On day 3 of coculture, cells were stimulated with $25 \mathrm{U} / \mathrm{ml} \mathrm{IL}-2$ or $10 \mathrm{ng} / \mathrm{ml} \mathrm{IL}-15$ for 25 minutes. $\mathrm{T}_{\mathrm{EM}}$ were then separated from the PCs, and were immediately fixed and permeabilized using BD Phosflow Fix Buffer I and Phosflow Perm Buffer III (BD Biosciences) according to manufacturer's instructions. After permeabilization, cells were stained with anti-STAT5 (pY694) (BD Bioscience, clone 47/Stat5pY694) and anti-CD8 (eBioscience, clone OKT8) conjugated primary antibodies for 30 minutes at $4^{\circ} \mathrm{C}$. Cells were washed twice with Stain Buffer (BD Bioscience) prior to acquisition on a BD LSR II flow cytometer.

\section{Assay of cytokine production by $\mathrm{T}_{\mathrm{EM}}$}

$\mathrm{CD}^{+}$or $\mathrm{CD}^{+} \mathrm{T}_{\mathrm{EM}}$ were cocultured with ECs or PCs that were either unstimulated or prestimulated with $50 \mathrm{ng} / \mathrm{ml} \mathrm{IFN}-\gamma$ for 72 hours using the same conditions as for the proliferation assays. After 24 hours of coculture, fresh supernatant was collected and assayed for IL-2 production using a DuoSet ELISA development system (R\&D Systems) according to the manufacturer's instructions.

\section{Genetic manipulations of vascular cells}

CIITA-transduced ECs and PCs were generated using a retroviral vector and characterized as previously described (44). In generation of shRNA-mediated knockdown of IDO1 in PCs, glycerol stocks of E. coli transformed with lentiviral shRNA vector plasmids (pSMART hCMV/TurboGFP, Dharmacon, GE Healthcare) containing either a target sequence for human IDO1 mRNA CCTGAAGACTGTAAGAAGT (IDO1-A) or TGTGCTCATTAGAGTCAAA (IDO1-B) or a nontargeting sequence were cotransfected with psPAX2 (produced by Didier Trono and available through AddGene, catalog 12260) and CMV VSV-G plasmids (produced by Robert Weinberg and available through AddGene, catalog 8454 ) into human $293 \mathrm{~T}$ cells (ATCC) using a calcium phoshate-based transfectant buffer (4 mg NaCl, $190 \mu \mathrm{g} \mathrm{KCl}, 50 \mu \mathrm{g} \mathrm{Na}_{2} \mathrm{HPO}_{4}, 2.5 \mathrm{mg}$ HEPES, $500 \mu \mathrm{g}$ glucose, $125 \mu \mathrm{mol} \mathrm{CaCl}$ in $500 \mu \mathrm{l} ; \mathrm{pH}$ 7.05). Lentiviral supernatant was collected at 48 hours and filtered through a $0.45-\mu \mathrm{m}$ filter. Polybrene $(8 \mu \mathrm{g} / \mathrm{ml}$; Sigma-Aldrich) was added to the supernatant that was subsequently used to transduce PCs for 8 hours. Following a 16-hour recovery period, a second 8-hour transduction was carried out, after which transduced PCs were selected with $1 \mu \mathrm{g} / \mathrm{ml}$ puromycin (Invitrogen) for 4 days. Successful shRNA-mediated knockdown was confirmed by qRT-PCR and immunoblotting. For siRNA knockdown of CEACAM-1, cells were transfected with 10 nM siRNA (control siRNA or CEACAM-1 s1978 siRNA, Ambion; control siRNA or CEACAM1 siRNA [J-009439-09-002], Dharmacon), as described previously (45). Successful siRNA-mediated knockdown was confirmed by immunoblotting.

\section{RNAseq analysis of vascular cells}

Confluent ECs and PCs grown on 12-well plates were stimulated or not with $50 \mathrm{ng} / \mathrm{ml}$ recombinant human IFN- $\gamma$ for 48 hours. Total RNA was purified using an RNeasy Mini kit (Qiagen), in which an on-column DNase treatment was included. Twelve strand-specific sequencing libraries, 3 replicates per condition, were produced from purified total RNA samples by the Illumina TruSeq stranded protocol. The libraries underwent 76-bp paired-end sequencing using an Illumina HiSeq 2500 according to Illumina protocols, generating an average of 35 million paired-end reads per library. For each read, we trimmed the first 6 nucleotides and the last nucleotide at the point where the Phred score of an examined base fell below 20 using in-house scripts. If, after trimming, the read was shorter than $45 \mathrm{bp}$, the whole read was discarded. Trimmed reads were mapped to the human reference genome (hg19) with a known transcriptome index (UCSC Known Gene annotation) with Tophat v2.1.1 (46) using the very-sensitive preset, first-strand library type, and providing the corresponding gene model annotation. Only the reads that mapped to a single unique location 
within the genome, with a maximum of 2 mismatches in the anchor region of the spliced alignment, were reported in these results. We used the default settings for all other Tophat options. Tophat alignments were then processed by Cuffdiff (Cufflinks v2.2.1; ref. 47) to obtain differential gene expression using first-strand library type, providing gene model annotation and the genome sequence file for detection and correction of sequence-specific bias that random hexamers can cause during library preparation. Sequencing data were deposited in NCBI's Gene Expression Omnibus (GEO) under accession number GSE109322.

\section{GSEA}

Gene sets from the Gene Ontology biological process (C5) were chosen for testing of enrichment of $\gamma$-PC versus $\gamma$-EC groups from RNAseq analysis. GSEA version 3.0 using the signal-to-noise ranking metric gene was used to analyze the data, and 100,000 permutations of the gene labels were used to test statistical significance. Results were visualized by computing enrichment score (ES), member position by the signalto-noise ranking metric (barcode), and ranking metric scale shown in output plots.

\section{qRT-PCR analysis}

To isolate RNA from ECs or PCs, cells were washed with sterile PBS and then processed using the RNeasy Mini kit, according to the manufacturer's protocol. The High-Capacity cDNA Reverse Transcription kit (Applied Biosystems) was used to synthesize cDNA from RNA. All qRT-PCR reactions were assembled with TaqMan gene expression master mix and predeveloped TaqMan gene expression probes (Applied Biosystems). Reactions were analyzed on a CFX96 real-time system using CFX Manager Software (Bio-Rad Laboratories). Gene expression levels were normalized to GAPDH.

\section{Western blot}

For Western blot analysis, EC or PC monolayers were washed in ice-cold PBS twice and lysed in RIPA buffer (Sigma-Aldrich). Samples were then mixed with Laemmli's sample buffer and heated at $95^{\circ} \mathrm{C}$ for 10 minutes prior to loading. After electrophoresis, samples were transferred onto a polyvinylidene difluoride membrane for 90 minutes at $4^{\circ} \mathrm{C}$. Membranes were blocked with TBST containing either $5 \%$ BSA or $5 \%$ nonfat dry milk, and anti-human IDO1 (Cell Signaling Technology, clone D5J4E) and anti-human CEACAM1 (Cell Signaling Technology, clone D3R8O), anti-human hsp90 (Sigma-Aldrich), or antihuman $\beta$-actin (Sigma-Aldrich) was added for overnight incubation at $4^{\circ} \mathrm{C}$. Bound antibodies were visualized with anti-mouse or anti-rabbit horseradish peroxidase (HRP)-conjugated antibodies (Thermo Fisher Scientific) and SuperSignal Femto or Pico West (Pierce).

\section{Tryptophan ELISA}

ECs and PCs were stimulated with IFN- $\gamma(50 \mathrm{ng} / \mathrm{ml})$ for 72 hours, after which the original media used for stimulation were removed and replaced with fresh RPMI media supplemented with $10 \%$ FBS. Forty-eight hours later, the conditioned media were collected for assessment of tryptophan level using the Tryptophan ELISA Kit (Novus) following manufacturer's instructions.

\section{Analysis of human skin grafts on immunodeficient mice}

Samples of normal adult human skin were obtained from discarded, deidentified surgical specimens through the Department of Pathology at Yale University. The superficial layers of the skin were harvested in 0.5-mm-thick sheets using a dermatome knife (gauge size, 0.016; Weck). The dermatomed skin was then divided into square-shaped pieces (approximately $1 \mathrm{~cm} \times 1 \mathrm{~cm}$ in size) and then stored in RPMI 1640 medium (Gibco) at $4^{\circ} \mathrm{C}$ until transplantation within the next few hours. Female 6- to 12 -week-old C.B-17 SCID/bg mice, purchased from Taconic Biosciences, were anesthetized with inhaled isoflurane cocktail via jar/nose cone, until there was no increase in respiratory rate or visible voluntary movement in response to pinching of the skin with forceps. Two $1-\mathrm{cm}^{2}$ mouse skin segments were removed from the upper back, and the defects were immediately covered with the human skin grafts and fixed with disposable staples $(3 \mathrm{M})$. The surgical procedure typically took 5 minutes ( \pm 1 minute) from start to finish and therefore isoflurane administered in a nose cone was sufficient. To minimize postoperative discomfort, a long-acting local anesthetic, bupivacaine $(0.25 \%)$, was injected intradermally and/or in the superficial subcutis radially out from the wound edges to approximately $0.5 \mathrm{~cm}$ immediately after surgery. Postsurgery analgesia was administered as meloxicam $0.3-1.0 \mathrm{mg} / \mathrm{kg}$ subcutaneously 24 hours and 48 hours after surgery. Animals 
were not used for experiments until at least 4 weeks after skin transplantation to allow proper healing. To initiate the experiment, 150 million PBMCs or saline control was administered through intraperitoneal injection, and the mice were euthanized and skin grafts harvested 7 days later. Harvested human skin samples were snap frozen in OCT and were sectioned at $5 \mu \mathrm{m}$ using a Microm cryostat. Sections were fixed in acetone for 10 minutes at $-20^{\circ} \mathrm{C}$, washed with PBS, and incubated with Dylight 649-labeled Ulex europeus agglutinin I (ULEX) (Vector Laboratories) along with anti-human $\alpha$-smooth muscle actin ( $\alpha$-SMA) (Sigma-Aldrich, clone 1A4; or Abcam, catalog number ab5694), anti-human CD3 (Abcam, catalog number ab5690), anti-human IDO1 (Cell Signaling Technology, clone D5J4E), and anti-human CEACAM1 (Abcam, clone CE1) diluted 1:100 in blocking solution (1\% BSA in PBS) overnight at $4^{\circ} \mathrm{C}$. To validate the IDO1 and CEACAM1 antibodies used for tissue staining, immunofluorescence microscopy was performed using shRNA IDO1-knockdown PCs and siRNA CEACAM1-knockdown PCs with respective controls, demonstrating antibody specificities (Supplemental Figure 7). Sections were washed with Tris-buffered saline, and then were incubated with appropriate Alexa Fluor-conjugated secondary antibodies in blocking solution (1:1,000 dilution) for 1 hour at room temperature. Sections were then washed with PBS, and coverslipped using ProLong Gold Mounting Reagent with DAPI (Life Technologies, P36935). Images were acquired using an Axiovert 200M microscopy system (Carl Zeiss MicroImaging) using Velocity software.

\section{Immunofluorescence staining of human renal biopsies}

Renal allograft biopsies analyzed in this study were collected at Addenbrooke's Hospital, with ethical approval by the hospital committee, diagnosed by V. Broecker, and then deidentified prior to analysis by immunofluorescence. Paraffin sections were dewaxed in xylene, boiled for 20 minutes in citrate buffer (10 $\mathrm{mM}, \mathrm{pH}$ 6.0) for antigen retrieval, and rehydrated. After washing 3 times with PBS, tissue sections were incubated with Dylight 649-labeled ULEX along with anti-human $\alpha$-SMA, anti-human IDO1, and antihuman CEACAM1 primary antibodies (clone numbers listed in previous section) diluted in blocking solution (1\% BSA in PBS) overnight at $4^{\circ} \mathrm{C}$ in a humidified chamber. Sections were washed 3 times with Trisbuffered saline, incubated with appropriate secondary antibodies diluted 1:1,000 in blocking solution for 1 hour at room temperature, washed again 3 times, and mounted on slides with ProLong Gold Mounting Reagent with DAPI. All immunofluorescence micrographs were acquired using an Axiovert 200M microscopy system. Images were captured using Velocity software.

\section{Quantification of immunofluorescence images}

Custom MATLAB code was used to quantify the expression of IDO1 or CEACAM1 by ECs and PCs in fluorescence microscopy images of human tissues (human skin grafts on immunodeficient mice and biopsy samples of acute renal allograft rejection). In brief, microvessels containing both ECs and PCs were first identified on the basis of combined ULEX and $\alpha$-SMA staining and images were cropped to these vessels to exclude irrelevant areas of the images from analysis. A programmatically generated threshold (using Otsu's method as described on the MathWorks website (https://www.mathworks.com/) was applied to distinguish both the $\mathrm{ULEX}^{+}(\mathrm{EC})$ and $\alpha-\mathrm{SMA}^{+}(\mathrm{PC})$ regions from background. Isolated areas of $\mathrm{ULEX}^{+} \alpha-\mathrm{SMA}^{-}(\mathrm{EC})$ or $\mathrm{ULEX}^{-} \alpha-\mathrm{SMA}^{+}$(PC) were next identified and $\mathrm{ULEX}^{+} \alpha-\mathrm{SMA}^{+}$double-positive overlap areas were excluded for analysis since expression of IDO1 or CEACAM1 in these areas could not be attributed to either cell type. Next, true IDO1 or CEACAM staining above background was identified by using a threshold determined from isotype stain control images for each of the tissues of interest. Finally, the degree of IDO1 or CEACAM1 colocalization to either ECs or PCs was determined by the relative area of each cell type positive for IDO1 or CEACAM1 (e.g., total area of IDO ${ }^{+} \alpha-\mathrm{SMA}^{+} \mathrm{ULEX}^{-}$divided by total area of $\alpha-\mathrm{SMA}^{+} \mathrm{ULEX}^{-}$). Statistical analyses were performed using either a Mann-Whitney $t$ test (copositive signal in PC-only regions for control vs. PBMC skins or time 0 vs. allograft-rejected kidneys) or a Wilcoxon matched-pairs signed-rank $t$ test (copositive signal in PC- versus EC-only regions in the same image of either PBMC skins or allograft-rejected kidneys).

\section{Statistics}

Data are expressed as mean \pm SEM. Statistical analyses were performed using GraphPad Prism software. Unpaired $t$ tests were used to make statistical comparisons between 2 groups. Comparisons between multiple groups were performed using 1-way ANOVA with Tukey's test for post hoc analysis. In all experiments, $P<0.05$ was considered statistically significant. 


\section{Study approval}

All protocols involving collection of and experimentation with human cells and tissues were approved by the Yale University Institutional Review Board. Renal biopsies analyzed in this study were collected at Addenbrooke's Hospital, with ethical approval by the hospital committee. All experiments involving animals were conducted according to protocols approved the Yale University Institutional Animal Care and Use Committee.

\section{Author contributions}

RL and JSP designed the research studies. RL, TDM, JM, LQ, GTT, FLG, CF, CX, PMC, NCKS, and DJW conducted experiments, acquired data, and/or analyzed data. VB collected and interpreted renal biopsies. RL and JSP wrote the manuscript.

\section{Acknowledgments}

This work is supported by NIH R01 HL051014 (to J.S. Pober and T.D. Manes). R. Liu was supported by the NIH National Research Service Award Predoctoral Fellowship F31-HL129563 and is currently supported by NIH Medical Scientist Training Program grant T32-GM007205. Identification and processing of renal biopsy specimens from Addenbrooke's Hospital for research was supported by funding from the NIHR Cambridge Biomedical Research Centre.

Address correspondence to: Jordan S. Pober, 10 Amistad Street, New Haven, Connecticut 06519, USA. Phone: 203.737.2292; Email: jordan.pober@yale.edu.

VB's present address is: Department of Clinical Pathology, Sahlgrenska University Hospital, Gothenburg, Sweden.

1. Heeger PS, et al. Pretransplant frequency of donor-specific, IFN-gamma-producing lymphocytes is a manifestation of immunologic memory and correlates with the risk of posttransplant rejection episodes. J Immunol. 1999;163(4):2267-2275.

2. Shiao SL, Kirkiles-Smith NC, Shepherd BR, McNiff JM, Carr EJ, Pober JS. Human effector memory CD4 $4^{+}$T cells directly recognize allogeneic endothelial cells in vitro and in vivo. J Immunol. 2007;179(7):4397-4404.

3. Lakkis FG, Lechler RI. Origin and biology of the allogeneic response. Cold Spring Harb Perspect Med. 2013;3(8):a014993.

4. Shiao SL, McNiff JM, Pober JS. Memory T cells and their costimulators in human allograft injury. J Immunol. 2005;175(8):4886-4896.

5. Lechler R, Ng WF, Steinman RM. Dendritic cells in transplantation--friend or foe? Immunity. 2001;14(4):357-368.

6. Manes TD, Pober JS, Kluger MS. Endothelial cell-T lymphocyte interactions: IP[corrected]-10 stimulates rapid transendothelial migration of human effector but not central memory $\mathrm{CD}^{+} \mathrm{T}$ cells. Requirements for shear stress and adhesion molecules. Transplantation. 2006;82(1 Suppl):S9-S14.

7. Proebstl D, et al. Pericytes support neutrophil subendothelial cell crawling and breaching of venular walls in vivo. J Exp Med. 2012;209(6):1219-1234.

8. Bose A, et al. Tumor-derived vascular pericytes anergize Th cells. J Immunol. 2013;191(2):971-981.

9. Maier CL, Pober JS. Human placental pericytes poorly stimulate and actively regulate allogeneic CD4 T cell responses. Arterioscler Thromb Vasc Biol. 2011;31(1):183-189.

10. Hart DN, Fuggle SV, Williams KA, Fabre JW, Ting A, Morris PJ. Localization of HLA-ABC and DR antigens in human kidney. Transplantation. 1981;31(6):428-433.

11. Pober JS, et al. Ia expression by vascular endothelium is inducible by activated T cells and by human gamma interferon. $J$ Exp Med. 1983;157(4):1339-1353.

12. Pober JS, Gimbrone MA. Expression of Ia-like antigens by human vascular endothelial cells is inducible in vitro: demonstration by monoclonal antibody binding and immunoprecipitation. Proc Natl Acad Sci USA. 1982;79(21):6641-6645.

13. Abrahimi $\mathrm{P}$, et al. Efficient gene disruption in cultured primary human endothelial cells by CRISPR/Cas9. Circ Res. 2015;117(2):121-128.

14. Abrahimi P, et al. Blocking MHC class II on human endothelium mitigates acute rejection. JCI Insight. 2016;1(1):e85293.

15. Epperson DE, Pober JS. Antigen-presenting function of human endothelial cells. Direct activation of resting CD8 T cells. J Immunol. 1994;153(12):5402-5412.

16. Alexander WS, Hilton DJ. The role of suppressors of cytokine signaling (SOCS) proteins in regulation of the immune response. Annu Rev Immunol. 2004;22:503-529.

17. Zarour HM. Reversing T-cell dysfunction and exhaustion in cancer. Clin Cancer Res. 2016;22(8):1856-1864.

18. Murray AG, Petzelbauer P, Hughes CC, Costa J, Askenase P, Pober JS. Human T-cell-mediated destruction of allogeneic dermal microvessels in a severe combined immunodeficient mouse. Proc Natl Acad Sci USA. 1994;91(19):9146-9150.

19. Maier CL, Shepherd BR, Yi T, Pober JS. Explant outgrowth, propagation and characterization of human pericytes. Microcirculation. 2010;17(5):367-380.

20. Rustenhoven J, Jansson D, Smyth LC, Dragunow M. Brain pericytes as mediators of neuroinflammation. Trends Pharmacol Sci. 
2017;38(3):291-304.

21. Weninger W, Biro M, Jain R. Leukocyte migration in the interstitial space of non-lymphoid organs. Nat Rev Immunol. 2014;14(4):232-246

22. Stark K, et al. Capillary and arteriolar pericytes attract innate leukocytes exiting through venules and 'instruct' them with pattern-recognition and motility programs. Nat Immunol. 2013;14(1):41-51.

23. Liu R, et al. IL-17 promotes neutrophil-mediated immunity by activating microvascular pericytes and not endothelium. $J$ Immunol. 2016;197(6):2400-2408.

24. Tu Z, et al. Retinal pericytes inhibit activated T cell proliferation. Invest Ophthalmol Vis Sci. 2011;52(12):9005-9010.

25. Su J, et al. Phylogenetic distinction of iNOS and IDO function in mesenchymal stem cell-mediated immunosuppression in mammalian species. Cell Death Differ. 2014;21(3):388-396.

26. Mezrich JD, Fechner JH, Zhang X, Johnson BP, Burlingham WJ, Bradfield CA. An interaction between kynurenine and the aryl hydrocarbon receptor can generate regulatory T cells. J Immunol. 2010;185(6):3190-3198.

27. Owe-Young R, et al. Kynurenine pathway metabolism in human blood-brain-barrier cells: implications for immune tolerance and neurotoxicity. J Neurochem. 2008;105(4):1346-1357.

28. Domev H, Milkov I, Itskovitz-Eldor J, Dar A. Immunoevasive pericytes from human pluripotent stem cells preferentially modulate induction of allogeneic regulatory T cells. Stem Cells Transl Med. 2014;3(10):1169-1181.

29. Kumar S, Wang J, Thomson AW, Gandhi CR. Hepatic stellate cells increase the immunosuppressive function of natural Foxp3 regulatory T cells via IDO-induced AhR activation. J Leukoc Biol. 2017;101(2):429-438.

30. Ferber IA, et al. Mice with a disrupted IFN-gamma gene are susceptible to the induction of experimental autoimmune encephalomyelitis (EAE). J Immunol. 1996;156(1):5-7.

31. Vermeire K, Heremans H, Vandeputte M, Huang S, Billiau A, Matthys P. Accelerated collagen-induced arthritis in IFN-gamma receptor-deficient mice. J Immunol. 1997;158(11):5507-5513.

32. Räisänen-Sokolowski A, Mottram PL, Glysing-Jensen T, Satoskar A, Russell ME. Heart transplants in interferon-gamma, inter leukin 4, and interleukin 10 knockout mice. Recipient environment alters graft rejection. J Clin Invest. 1997;100(10):2449-2456

33. Konieczny BT, et al. IFN-gamma is critical for long-term allograft survival induced by blocking the CD28 and CD40 ligand T cell costimulation pathways. J Immunol. 1998;160(5):2059-2064.

34. Halloran PF, et al. Interferon-gamma acts directly on rejecting renal allografts to prevent graft necrosis. Am J Pathol. 2001;158(1):215-226.

35. Mele TS, et al. IFN-gamma is an absolute requirement for spontaneous acceptance of liver allografts. Am J Transplant. 2003;3(8):942-951.

36. Miura M, El-Sawy T, Fairchild RL. Neutrophils mediate parenchymal tissue necrosis and accelerate the rejection of complete major histocompatibility complex-disparate cardiac allografts in the absence of interferon-gamma. Am J Pathol. 2003;162(2):509-519.

37. Ohmori Y, Hamilton TA. IFN-gamma selectively inhibits lipopolysaccharide-inducible JE/monocyte chemoattractant protein-1 and $\mathrm{KC} / \mathrm{GRO} /$ melanoma growth-stimulating activity gene expression in mouse peritoneal macrophages. J Immunol. 1994;153(5):2204-2212.

38. Luckraz H, et al. Microvascular changes in small airways predispose to obliterative bronchiolitis after lung transplantation J Heart Lung Transplant. 2004;23(5):527-531.

39. Luckraz H, Goddard M, McNeil K, Atkinson C, Sharples LD, Wallwork J. Is obliterative bronchiolitis in lung transplantation associated with microvascular damage to small airways? Ann Thorac Surg. 2006;82(4):1212-1218.

40. Bishop GA, Waugh JA, Landers DV, Krensky AM, Hall BM. Microvascular destruction in renal transplant rejection. Transplantation. 1989;48(3):408-414.

41. Matsumoto Y, McCaughan GW, Painter DM, Bishop GA. Evidence that portal tract microvascular destruction precedes bile duct loss in human liver allograft rejection. Transplantation. 1993;56(1):69-75.

42. Bruneau S, et al. Key features of the intragraft microenvironment that determine long-term survival following transplantation. Front Immunol. 2012;3:54

43. Thornton SC, Mueller SN, Levine EM. Human endothelial cells: use of heparin in cloning and long-term serial cultivation. Science. 1983;222(4624):623-625.

44. Manes TD, Pober JS. Antigen presentation by human microvascular endothelial cells triggers ICAM-1-dependent transendothelial protrusion by, and fractalkine-dependent transendothelial migration of, effector memory CD4+ T cells. J Immunol. 2008;180(12):8386-8392

45. Manes TD, Pober JS. Identification of endothelial cell junctional proteins and lymphocyte receptors involved in transendothelial migration of human effector memory CD4 ${ }^{+}$T cells. J Immunol. 2011;186(3):1763-1768.

46. Trapnell C, Pachter L, Salzberg SL. TopHat: discovering splice junctions with RNA-Seq. Bioinformatics. 2009;25(9):1105-1111

47. Trapnell C, et al. Transcript assembly and quantification by RNA-Seq reveals unannotated transcripts and isoform switching during cell differentiation. Nat Biotechnol. 2010;28(5):511-515 